\title{
Cubic-interaction-induced deformations of higher-spin symmetries
}

\author{
Euihun Joung $^{a}$ and Massimo Taronna ${ }^{b}$ \\ ${ }^{a}$ AstroParticule et Cosmologie ${ }^{1}$ \\ 10 rue Alice Domon et Léonie Duquet, 75205 Paris Cedex 13, France \\ ${ }^{b}$ Max-Planck-Institut für Gravitationsphysik (Albert-Einstein-Institut) \\ Am Mühlenberg 1, 14476 Golm, Germany \\ E-mail: joung@apc.univ-paris7.fr, massimo.taronna@aei.mpg.de
}

\begin{abstract}
The deformations of higher-spin symmetries induced by cubic interactions of symmetric massless bosonic fields are analyzed within the metric-like formalism. Our analysis amends the existing classification according to gauge-algebra deformations taking into account also gauge-transformation deformations. In particular, we identify a class of couplings which leave the gauge algebra Abelian but deform one (out of three) gauge transformation, and another class of couplings which deform all three gauge transformations in (A)dS but only two in the flat-space limit. The former class is related to higher-spin algebra multiplets (representations of the global algebra) together with the massless-massive-massive couplings which we also briefly discuss. The latter class is what makes (A)dS a distinguished background for higher-spin interactions and includes in particular the gravitational interactions of higher-spin fields, retrospectively accounting for the Fradkin-Vasiliev solution to the Aragone-Deser problem. We also study the restriction of gauge symmetries to global symmetries (higher-spin algebra) discussing the invariant bilinear form and the cyclicity of the structure constants. A possible generalization of the analysis to partially-massless fields is also commented.
\end{abstract}

KeYwords: Gauge Symmetry, Global Symmetries, AdS-CFT Correspondence

ARXIV EPRINT: 1311.0242

\footnotetext{
${ }^{1}$ UMR 7164 (CNRS, Université Paris 7, CEA, Observatoire de Paris).
} 


\section{Contents}

1 Introduction $\quad 2$

1.1 General procedure 2

1.2 Summary of our results 4

$\begin{array}{lll}1.3 & \text { Organization of paper } & 6\end{array}$

2 Review: cubic interactions $\quad 6$

3 Deformations of gauge transformations and non-deforming couplings $\quad 8$

3.1 Deformations of gauge transformations 8

$\begin{array}{lll}3.2 & \text { Non-deforming couplings } & 10\end{array}$

4 Deformations of gauge algebras and abelian couplings 16

$\begin{array}{ll}4.1 \text { Deformations of gauge algebras } & 16\end{array}$

$\begin{array}{lll}4.2 & \text { Abelian interactions } & 16\end{array}$

$\begin{array}{lll}5 & \text { Global symmetries } & \mathbf{1 7}\end{array}$

$\begin{array}{lll}5.1 & \text { Killing tensors } & 17\end{array}$

$\begin{array}{lll}5.2 & \text { Higher-spin algebras } & 18\end{array}$

6 Abelian and non-deforming interactions as $H$-couplings 25

6.1 Contraction of curls 25

$6.2 H$-couplings 26

$\begin{array}{lll}6.3 & \text { Examples } & 29\end{array}$

$\begin{array}{lll}7 & \text { Discussions } & 30\end{array}$

$\begin{array}{ll}\text { A Transverse and traceless part } & 33\end{array}$

$\begin{array}{ll}\text { B Constants generated by } \lambda & 34\end{array}$

C Analysis of trivial deformations

$\begin{array}{ll}\text { D Counting of }(\mathrm{A}) \mathrm{dS} \text { structure constants } & 36\end{array}$

E No-go for the spin-3 gravitational coupling in flat space $\quad 37$ 


\section{Introduction}

Constructing a theory of interacting higher-spin (HS) fields encounters several surprises, such as higher derivatives, unphysical degrees of freedom or acausality - novel obstacles which do not arise so often in their lower-spin cousins. ${ }^{1}$ On the other hand, there exist two examples of HS theories - String theory and Vasiliev's equations [2-6] — which so far have proved to be safe from such problems. However, the mechanisms making them bypass such difficulties, or more generally, the systematics of consistent HS interactions, are not fully understood yet. For these reasons, we find it important and challenging to explore this systematics in order to attain a better understanding of String theory and Vasiliev's equations, as well as to uncover possible links between them or, eventually, new theories of HS fields.

Relatively well-understood nowadays is the systematics of HS cubic interactions. ${ }^{2}$ As regards the case of massless HS bosons of symmetric type, the exhaustive list of flat-space cubic interactions has been identified in $[7,8]$, and classified according to the corresponding gauge-algebra deformations in [25]. About (A)dS cubic interactions, all non-Abelian vertices have been constructed in [20,21, 41] in the frame-like approach (see also [23]). In our previous works [42-47], we have constructed all (A)dS cubic interactions in the metric-like approach, ${ }^{3}$ but without touching the issues of their gauge-symmetry deformations. In this paper, we analyze how the previously constructed cubic interactions induce deformations of the gauge transformations and gauge algebras. This reveals some interesting structures of HS gauge interactions. For instance, although the total number of consistent $s_{1}-s_{2}-s_{3}$ couplings is the same in flat and in (A)dS spaces, the corresponding deformation of gauge symmetries is qualitatively different. Postponing a detailed account to the next sections, let us just comment that this is a general mechanism behind the Fradkin-Vasiliev construction $[20,21]$ where (A)dS backgrounds play a distinguished role.

\subsection{General procedure}

For a more concrete understanding, let us remind the general program [49]. The starting point is the expansion of the sought action and gauge transformations in powers of the fields:

$$
S=S^{(2)}+S^{(3)}+\cdots, \quad \delta_{\varepsilon} \varphi=\delta_{\varepsilon}^{(0)} \varphi+\delta_{\varepsilon}^{(1)} \varphi+\cdots .
$$

Here, the superscript $(n)$ means that the corresponding term involves $n$-th powers of the fields. In this expansion scheme, the gauge invariance of the action is recast into an infinite

\footnotetext{
${ }^{1}$ See e.g. [1] (and references therein) for a review about such obstructions and their possible resolutions.

${ }^{2}$ Many efforts have been devoted to this direction, among which let us mention e.g. the light-cone approach of [7-11], the metric-like approach of [12-19], the frame-like approach of [20-23], the BRST-BV constructions of [24-31], etc. Other relevant works are e.g. [32-36].

Beyond the cubic order, much less is known, in particular, about the (non-)local nature of the corresponding Lagrangians and most importantly about unitarity. See [37, 38] in the light-cone formalism and [39, 40] for more recent results on quartic interactions.

${ }^{3}$ Our construction covers also massless, partially-massless and massive spectrum. Note that when considering partially-massless and massive fields, we have employed the gauge consistency at the level of traceless and transverse vertices, which turn out to be equivalent to the requirement of Stueckelberg invariance. These results agree with those obtained in [48], while the study of the complete dynamics was left untouched.
} 
number of coupled equations:

$$
\delta_{\varepsilon} S=0 \Rightarrow\left\{\begin{array}{c}
\delta_{\varepsilon}^{(0)} S^{(2)}=0 \\
\delta_{\varepsilon}^{(0)} S^{(3)}+\delta_{\varepsilon}^{(1)} S^{(2)}=0 \\
\delta_{\varepsilon}^{(0)} S^{(4)}+\delta_{\varepsilon}^{(1)} S^{(3)}+\delta_{\varepsilon}^{(2)} S^{(2)}=0 \\
\vdots
\end{array}\right.
$$

where $S^{(2)}$ and $\delta_{\varepsilon}^{(0)} \varphi$ are the free action and the corresponding gauge transformations, respectively. Higher-order parts of the action, $S^{(n \geq 3)}$, and higher-order gauge transformations, $\delta_{\varepsilon}^{(n \geq 1)} \varphi$, can be identified starting from $S^{(2)}$ and $\delta_{\varepsilon}^{(0)} \varphi$ by solving the above equations. The strategy is to solve the equations (i) of (1.2) in two steps as

$$
S^{(2)}, \delta_{\varepsilon}^{(0)} \varphi \stackrel{(1)}{\longrightarrow} S^{(3)} \stackrel{(1)}{\longrightarrow} \delta_{\varepsilon}^{(1)} \varphi \stackrel{\text { (2) }}{\longrightarrow} S^{(4)} \stackrel{(2)}{\longrightarrow} \delta_{\varepsilon}^{(2)} \varphi \longrightarrow \cdots,
$$

where (i) represents the same condition as (1) but solved this time on the shell of free EoM. In particular, at each order one can first solve for $S^{(n+2)}$ using (in and then read off $\delta_{\varepsilon}^{(n)} \varphi$ from (n). On the other hand, the full non-linear gauge transformations must form an (open) algebra:

$$
\delta_{\varepsilon_{1}} \delta_{\varepsilon_{2}} \varphi-\delta_{\varepsilon_{2}} \delta_{\varepsilon_{1}} \varphi=\delta_{\llbracket \varepsilon_{1}, \varepsilon_{2} \rrbracket} \varphi+(\text { trivial }),
$$

where (trivial) refers to the trivial symmetry ${ }^{4}$ of the action, while the commutator $\llbracket \varepsilon_{1}, \varepsilon_{2} \rrbracket$ is in principle field-dependent and can be expanded as

$$
\llbracket \varepsilon_{1}, \varepsilon_{2} \rrbracket=\llbracket \varepsilon_{1}, \varepsilon_{2} \rrbracket^{(0)}+\llbracket \varepsilon_{1}, \varepsilon_{2} \rrbracket^{(1)}+\cdots .
$$

For the purpose of the present letter, we focus on the lowest-order part of the commutator:

$$
\delta_{\varepsilon_{1}}^{(0)} \delta_{\varepsilon_{2}}^{(1)} \varphi-\delta_{\varepsilon_{2}}^{(0)} \delta_{\varepsilon_{1}}^{(1)} \varphi=\delta_{\llbracket \varepsilon_{1}, \varepsilon_{2} \rrbracket}^{(0)} \rrbracket^{(0)} \varphi
$$

which is field-independent and can be entirely obtained from $\delta_{\varepsilon}^{(1)}$. To sum up, once consistent cubic interactions are determined for a given free theory, then they induce deformations of the gauge transformations and also of the gauge algebra:

$$
S^{(3)} \Rightarrow \delta_{\varepsilon}^{(1)} \varphi \Rightarrow \llbracket \varepsilon_{1}, \varepsilon_{2} \rrbracket^{(0)} .
$$

For the analysis of such deformations, we need first to define the free theory. In the following, we shall consider symmetric massless bosonic fields in a constant curvature background, namely (A)dS.

Ambient-space formulation and Transverse and Traceless part. In order to conveniently treat fields in (A)dS, we use the ambient-space formulation where fields $\varphi_{\mu_{1} \cdots \mu_{s}}(x)$ are described through the corresponding ambient avatars $\Phi_{M_{1} \cdots M_{s}}(X)$, defined in a $(d+1)$ dimensional flat space and subject to homogeneity and tangentiality conditions:

$$
\left(X \cdot \partial_{X}-U \cdot \partial_{U}+2+\mu\right) \Phi(X, U)=0, \quad X \cdot \partial_{U} \Phi(X, U)=0 .
$$

\footnotetext{
${ }^{4}$ A trivial symmetry of an action $S$ is given in de-Witt notation by $\delta \varphi^{i}=C^{i j} \delta S / \delta \varphi^{j}$ with $C^{i j}=-C^{j i}$, and is proportional to the EoM by definition.
} 
The mass of the field is here parametrized by the degree of homogeneity $\mu$, and when the field is massless (that is $\mu=0$ ), it admits gauge symmetries:

$$
\delta_{E}^{(0)} \Phi=U \cdot \partial_{X} E \quad\left[\partial_{U}^{2} E=0,\left(X \cdot \partial_{X}-U \cdot \partial_{U}\right) E=0, X \cdot \partial_{U} E=0\right] .
$$

In constructing gauge-invariant interaction vertices, we focus for simplicity on the transverse and traceless (TT) part of the latter, disregarding the terms proportional to divergences and traces of the fields. This is equivalent to consider, instead of the full vertices and their gauge variations, their quotient modulo the following equivalence relations:

$$
\partial_{U} \cdot \partial_{X} \Phi \stackrel{\mathrm{TT}}{=} 0, \quad \partial_{U}^{2} \Phi \stackrel{\mathrm{TT}}{=} 0 ; \quad \partial_{U} \cdot \partial_{X} E \stackrel{\mathrm{TT}}{=} 0, \quad \partial_{X}^{2} E \stackrel{\mathrm{TT}}{=} 0 .
$$

In this setting, the free action assumes a general form and is given simply by

$$
S^{(2)}[\Phi] \stackrel{\mathrm{TT}}{=}-\left.\frac{1}{2} \int_{(\mathrm{A}) \mathrm{d} S} e^{\partial_{U_{1}} \cdot \partial_{U_{2}}} \Phi\left(X, U_{1}\right) \partial_{X}^{2} \Phi\left(X, U_{2}\right)\right|_{U_{i}=0},
$$

and the interaction parts of action $S^{(n \geq 3)}[\Phi]$ together with the corresponding gauge transformations $\delta_{E}^{(n \geq 1)} \Phi$ can be also studied within this description.

Let us emphasize that here we do not impose any gauge condition on the theory, but we merely attempt to identify the TT part of interacting vertices - that is the reason why we introduce the notion of the equivalence class $\stackrel{\text { TT }}{=}$. The point is, as shown in [44] and also recalled in the appendix A, that it is possible to determine such TT part without having any information on the other parts of the vertices.

\subsection{Summary of our results}

The interaction parts of the action can be conveniently expressed as

$$
\left.S^{(n)}[\Phi] \stackrel{\mathrm{TT}}{=} \int_{(\mathrm{A}) \mathrm{dS}} C^{(n)} \Phi\left(X_{1}, U_{1}\right) \cdots \Phi\left(X_{n}, U_{n}\right)\right|_{\substack{X_{i}=X \\ U_{i}=0}}
$$

in terms of a differential operator $C^{(n)}$ in $X_{i}$ and $U_{i}$. Each operator $C^{(n)}$ is constrained by the gauge-invariance conditions (1.2). For cubic interactions $(n=3)$, the general solution to them was found to be

$$
C^{(3)}=\sum_{s_{1}, s_{2}, s_{3}, n} k_{n}^{s_{1} s_{2} s_{3}} P_{s_{1} s_{2} s_{3}}^{[n]}, \quad P_{s_{1} s_{2} s_{3}}^{[n]}=e^{\lambda \mathcal{D}} Y_{1}^{s_{1}-n} Y_{2}^{s_{2}-n} Y_{3}^{s_{3}-n} G^{n},
$$

where the $s_{i}$ 's are the spins of the fields involved in the interactions and $n=0,1, \ldots, s_{\min }$ labels the possible interactions for a given set of fields. The differential operator $\mathcal{D}$ acting on $Y_{i}$ 's and $G$ generates the curvature (treated here as $\lambda$ ) corrections, and its form will be provided later. The $Y_{i}$ 's and $G$ are given by

$$
Y_{i}=\partial_{U_{i}} \cdot \partial_{X_{i+1}}, \quad G=\partial_{U_{1}} \cdot \partial_{U_{2}} \partial_{U_{3}} \cdot \partial_{X_{1}}+\partial_{U_{2}} \cdot \partial_{U_{3}} \partial_{U_{1}} \cdot \partial_{X_{2}}+\partial_{U_{3}} \cdot \partial_{U_{1}} \partial_{U_{2}} \cdot \partial_{X_{3}},
$$

and they represent different tensor structures allowed by gauge invariance. 


\begin{tabular}{|c|c|c|c|c|c|c|}
\hline & $n$ & $\# \partial$ & $\delta_{E_{1}}^{(1)}$ & $\delta_{E_{2}}^{(1)}$ & $\delta_{E_{3}}^{(1)}$ & $C^{(3)}$ \\
\hline \multirow{3}{*}{ Class I } & 0 & $s_{1}+s_{2}+s_{3}$ & $=0$ & $=0$ & $=0$ & \multirow{4}{*}{$\begin{array}{c}\approx \tilde{K}\left(Y_{\ell}, H_{12}, H_{23}, H_{31}\right) \\
\quad \ell=2 \text { or } 3\end{array}$} \\
\hline & $\vdots$ & $\vdots$ & $\vdots$ & $\vdots$ & $\vdots$ & \\
\hline & $\frac{s_{2}+s_{3}-s_{1}}{-\frac{2}{-2}}$ & $2 s_{1}$ & $=0$ & $\vdots$ & $\vdots$ & \\
\hline & $\vdots$ & $\vdots$ & $\neq 0$ & $\vdots$ & $\vdots$ & \\
\hline \multirow[t]{2}{*}{ Class II } & $\vdots$ & $\vdots$ & $\vdots$ & $\vdots$ & $\vdots$ & \multirow[t]{2}{*}{$\approx \tilde{K}\left(Y_{1}, H_{12}, H_{23}, H_{31}\right)$} \\
\hline & $\frac{s_{3}+s_{1}-s_{2}}{-2}$ & $2 s_{2}$ & $\vdots$ & $=0$ & $=0$ & \\
\hline \multirow{3}{*}{ Class III } & $\vdots$ & $\vdots$ & $\vdots$ & $\neq 0$ & $\Lambda$ & \multirow{6}{*}{$-----------{ }_{-}$} \\
\hline & $\vdots$ & $\vdots$ & $\vdots$ & $\vdots$ & $\vdots$ & \\
\hline & $\frac{s_{1}+s_{2}-s_{3}}{--\underline{2}--}$ & $2 s_{3}$ & $\vdots$ & $\vdots$ & $\Lambda$ & \\
\hline \multirow{3}{*}{ Class IV } & $\vdots$ & $\vdots$ & $\vdots$ & $\vdots$ & $\neq 0$ & \\
\hline & $\vdots$ & $\vdots$ & $\vdots$ & $\vdots$ & $\vdots$ & \\
\hline & $s_{3}$ & $s_{1}+s_{2}-s_{3}$ & $\neq 0$ & $\neq 0$ & $\neq 0$ & \\
\hline
\end{tabular}

Table 1. Classification of cubic interactions $\left(s_{1} \geq s_{2} \geq s_{3}\right)$ according to the deformations of gauge transformations. Here $\#_{\partial}$ is the number of (highest, in the (A)dS case) derivatives involved in $C^{(3)}$. Notice that depending on the choice of $s_{1}-s_{2}-s_{3}$, some of the above classes can be empty.

Each of the cubic interactions $P_{s_{1} s_{2} s_{3}}^{[n]}$ induces a deformation of gauge transformation $\delta_{E}^{(1)} \Phi$ and gauge algebra $\llbracket E_{1}, E_{2} \rrbracket^{(0)}$. However, the main question is to analyze if those are trivial, being a mere consequence of gauge-field and/or gauge-parameter redefinitions. Hence, in order to identify the non-trivial ones, we need to analyze the effects of redefinitions on the deformations of gauge symmetries. ${ }^{5}$ The latter analysis is one of the main contents of the present article, and the results are summarized in table 1. An important lesson is that cubic interactions corresponding to trivial deformations of gauge symmetries are related to the appearance of a new tensor structure $H_{i j}$ :

$$
H_{i j}=\partial_{U_{i}} \cdot \partial_{X_{j}} \partial_{U_{j}} \cdot \partial_{X_{i}}-\partial_{X_{i}} \cdot \partial_{X_{j}} \partial_{U_{i}} \cdot \partial_{U_{j}} \cdot
$$

The $H_{i j}$ 's are operators taking the curls of the $i$-th and $j$-th fields and contracting them. They are gauge invariant without making use of the on-shell condition, and hence, they do not lead to any deformation of the gauge transformations. It turns out that for $s_{1} \geq s_{2} \geq s_{3}$ the couplings can be organized as follows:

- Class $I$ : the couplings which can be re-expressed as a function of $H_{i j}$ 's and $Y_{2}$ (or $\left.Y_{3}\right)$ do not deform any of the gauge transformations $\delta_{E_{1}}^{(1)}, \delta_{E_{2}}^{(1)}$ and $\delta_{E_{3}}^{(1)}$;

- Class II : the couplings which can be re-expressed as a function of $H_{i j}$ 's and $Y_{1}$ do not deform the gauge transformations $\delta_{E_{2}}^{(1)}$ and $\delta_{E_{3}}^{(1)}$ but $\delta_{E_{1}}^{(1)}$;

\footnotetext{
${ }^{5}$ By gauge symmetries, we shall refer to both gauge transformations and gauge algebras.
} 
- Class III $\&$ IV : the couplings which cannot be re-expressed as a function of $H_{i j}$ 's as above always deform all the gauge transformations in (A)dS (in flat space, the couplings belonging to Class III deform two of the gauge transformations while those belonging to Class IV deform all of them).

As regards the gauge-algebra deformations, the classification can be stated as follows:

- Gauge algebra: the deformation of the bracket $\llbracket E_{1}, E_{2} \rrbracket_{3}^{(0)}$ is non-trivial if and only if both of $\delta_{E_{1}}^{(1)}$ and $\delta_{E_{2}}^{(1)}$ are non-trivial.

Let us remark that the couplings of Class II make the fields $\Phi_{2}$ and $\Phi_{3}$ charged with respect to $\Phi_{1}$, so they are relevant for HS-algebra multiplets and in general for the classification of representations of HS algebra. The couplings of Class III induce non-trivial deformations $\delta_{E_{3}}^{(1)}$, but it vanishes in the flat-space limit. This class includes in particular the lowestderivative $s-s-2$ couplings, namely the gravitational interactions of HS fields: in (A)dS, the corresponding $\delta_{E_{3}}^{(1)}$ reproduces the general coordinate covariance for HS fields, while in the flat-space limit, the latter covariance is lost, so the spin 2 field is found to not couple gravitationally to HS fields. Therefore, one recognizes the Aragone-Deser problem [50] and the Fradkin-Vasiliev solution [20, 21] in this framework.

Since any cubic interaction can be expressed in terms of a coupling between a gauge field and a current, it may be also useful to restate the classification above in terms of currents. In both Class I and II cases, the corresponding currents are gauge invariant being bilinear in the HS curvature tensors: generalized Bell-Robinson currents. More in details, they are improvements or genuine Noether currents depending on whether the corresponding couplings belong to Class I or II. On the other hand, the currents associated with the couplings belonging to Class III-IV are not gauge invariant. Let us also note that the couplings belonging to Class I, II and III-IV correspond respectively to the Abelian, current and non-Abelian couplings studied in [41] within the frame-like formalism.

\subsection{Organization of paper}

The paper is organized as follows. In section 2 we review the construction of metric-like HS cubic interactions. In section 3 we analyze the non-trivial deformations of gaugetransformation deformations arriving to table 1 . In section 4 we analyze the gauge-algebra deformations, while in section 5 we consider their restriction to global symmetries. In section 6 we give some details on the explicit structure of the non-deforming couplings in (A)dS. Our conclusions, together with discussions on the partially-massless extension of the classification, are presented in section 7 . The appendices contain some technical details.

\section{Review: cubic interactions}

For completeness and in order to fix the notations, we briefly summarize our previous results. For cubic interactions, that is the $n=3$ case of (1.12), the ansatz ${ }^{6}$ can be further

\footnotetext{
${ }^{6}$ Here, we use the convention that all the fields entering in the interactions are treated as different fields, so that no symmetry under field-label interchange is assumed. This convention makes more transparent
} 
simplified, removing the ambiguities of integration by parts and field redefinitions, as

$$
\left.S^{(3)}\left[\Phi_{1}, \Phi_{2}, \Phi_{3}\right] \stackrel{\text { TT }}{=} \int_{(\mathrm{A}) \mathrm{d} S} C(Y, Z) \Phi_{1}\left(X_{1}, U_{1}\right) \Phi_{2}\left(X_{2}, U_{2}\right) \Phi_{3}\left(X_{3}, U_{3}\right)\right|_{\substack{X_{i}=X \\ U_{i}=0}},
$$

where $C(Y, Z)$ is a polynomial function of six variables:

$$
Y_{i}:=\partial_{U_{i}} \cdot \partial_{X_{i+1}}, \quad Z_{i}:=\partial_{U_{i+1}} \cdot \partial_{U_{i-1}} \quad[i \simeq i+3] .
$$

When one of the fields, say $\Phi_{1}$, is massless, the cubic interaction must be compatible with the corresponding gauge symmetry: $\delta_{E_{1}}^{(0)} S^{(3)}+\delta_{E_{1}}^{(1)} S^{(2)}=0$. This condition implies a weaker condition: $\delta_{E_{1}}^{(0)} S^{(3)} \approx 0$, where $\approx$ is henceforth the equivalence modulo free field equations as well as traces and divergences. The latter condition can be translated into the following differential equation:

$$
\left[Y_{2} \partial_{Z_{3}}-Y_{3} \partial_{Z_{2}}-\lambda\left(Y_{2} \partial_{Y_{2}}-Y_{3} \partial_{Y_{3}}+\frac{\mu_{2}-\mu_{3}}{2}\right) \partial_{Y_{1}}\right] C(Y, Z)=0
$$

Here, $\lambda$ is an auxiliary variable introduced to simplify the computation: it eventually generates the cosmological constant $\Lambda$ with some dimension dependent factor (see appendix B for more details).

In the case of cubic interactions, where all three fields are massless: $\mu_{1}=\mu_{2}=\mu_{3}=0$, the general solution to the cubic-interaction problem is given by

$$
C(Y, Z)=\left.e^{\lambda \mathcal{D}} K\left(Y_{1}, Y_{2}, Y_{3}, G\right)\right|_{G=G(Y, Z)},
$$

where $\mathcal{D}$ and $G(Y, Z)$ are defined by

$$
\begin{aligned}
\mathcal{D} & :=Z_{1} \partial_{Y_{2}} \partial_{Y_{3}}+Z_{1} Z_{2} \partial_{Y_{3}} \partial_{G}+\text { cyc. }+Z_{1} Z_{2} Z_{3} \partial_{G}^{2}, \\
G(Y, Z) & :=Y_{1} Z_{1}+Y_{2} Z_{2}+Y_{3} Z_{3} .
\end{aligned}
$$

From the solution (2.4), one can extract the $s_{1}-s_{2}-s_{3}$ interactions by Taylor-expanding the function $K$, and the contribution of each monomial in $K$ to the cubic interaction $C$ reads (for $n=0,1, \ldots, s_{\min }$ )

$$
\begin{aligned}
P_{s_{1} s_{2} s_{3}}^{[n]}(Y, Z) & :=\left.e^{\lambda \mathcal{D}} Y_{1}^{s_{1}-n} Y_{2}^{s_{2}-n} Y_{3}^{s_{3}-n} G^{n}\right|_{G=G(Y, Z)} \\
& =Y_{1}^{s_{1}-n} Y_{2}^{s_{2}-n} Y_{3}^{s_{3}-n}[G(Y, Z)]^{n}+\mathcal{O}(\lambda) .
\end{aligned}
$$

The first term in the second line corresponds to the highest-derivative part of coupling while $\mathcal{O}(\lambda)$ represents the lower-derivative terms. So, the highest number, $\#_{\partial}$, of derivatives in $P_{s_{1} s_{2} s_{3}}^{[\lambda]}$ is

$$
\#_{\partial}=s_{1}+s_{2}+s_{3}-2 n,
$$

and the corresponding part coincides with the flat-space coupling in the $\Lambda \rightarrow 0$ limit.

the analysis of the present work, while it is equivalent to the convention of a single generating function of HS fields. Hence, the results obtained in this convention can also account for the self-interaction cases. 


\section{Deformations of gauge transformations and non-deforming couplings}

\subsection{Deformations of gauge transformations}

After constructing all consistent cubic interactions $S^{(3)}$, the next step is to identify the corresponding deformations of the gauge transformations $\delta_{E_{i}}^{(1)}$ from the gauge-invariance condition:

$$
\delta_{E_{1}}^{(1)}\left(S^{(2)}\left[\Phi_{2}\right]+S^{(2)}\left[\Phi_{3}\right]\right)+\delta_{E_{1}}^{(0)} S^{(3)}\left[\Phi_{1}, \Phi_{2}, \Phi_{3}\right]=0 .
$$

Here, without loss of generality, we have chosen $E_{i}=E_{1}$. Using the form (1.11) of the quadratic action, the above condition can be put into

$$
\stackrel{\delta_{E_{1}}^{(0)} S^{(3)}\left[\Phi_{1}, \Phi_{2}, \Phi_{3}\right]}{\stackrel{\text { TT }}{=} \int_{(\mathrm{A}) \mathrm{dS}} e^{\partial_{U_{1}} \cdot \partial_{U_{2}}}\left[\delta_{E_{1}}^{(1)} \Phi_{2}\left(X, U_{1}\right) \partial_{X}^{2} \Phi_{2}\left(X, U_{2}\right)+\delta_{E_{1}}^{(1)} \Phi_{3}\left(X, U_{1}\right) \partial_{X}^{2} \Phi_{3}\left(X, U_{2}\right)\right]_{U_{i}=0} .}
$$

As one can see from this expression, the deformation $\delta_{E_{1}}^{(1)}$ can be obtained by computing the off-shell variation of the cubic interactions (2.1). The latter reads

$$
\begin{aligned}
& \delta_{E_{1}}^{(0)} S^{(3)}\left[\Phi_{1}, \Phi_{2}, \Phi_{3}\right] \\
& \left.\stackrel{\mathrm{TT}}{=} \int_{(\mathrm{A}) \mathrm{d} \mathrm{S}} \partial_{Y_{1}} C(Y, Z) E_{1}\left(X_{1}, U_{1}\right) \frac{1}{2}\left(\partial_{X_{3}}^{2}-\partial_{X_{2}}^{2}\right) \Phi_{2}\left(X_{2}, U_{2}\right) \Phi_{3}\left(X_{3}, U_{3}\right)\right|_{\substack{X_{i}=X \\
U_{i}=0}},
\end{aligned}
$$

where $C$ is the solution given in eq. (2.4). In order to obtain the deformations $\delta_{E_{1}}^{(1)} \Phi_{2}$ and $\delta_{E_{1}}^{(1)} \Phi_{3}$, we need to recast eq. (3.3) into the form of eq. (3.2). More precisely, we massage the terms proportional to $\partial_{X_{3}}^{2}$ and $\partial_{X_{2}}^{2}$ in eq. (3.3) to get the expressions for $\delta_{E_{1}}^{(1)} \Phi_{3}$ and $\delta_{E_{1}}^{(1)} \Phi_{2}$, respectively. Herefrom, let us focus on $\delta_{E_{1}}^{(1)} \Phi_{3}$, because $\delta_{E_{1}}^{(1)} \Phi_{2}$ can be obtained from the former by interchanging the field labels. What one needs to do is to integrate by parts all the $\partial_{X_{3}}$ 's in $\partial_{Y_{1}} C(Y, Z)$ of eq. (3.3) because in (3.2) there is no derivative acting on $\partial_{X}^{2} \Phi_{3}$. Notice that, since the variable $Y_{2}$ involves $\partial_{X_{3}}$, we can no more use such a variable in $C(Y, Z)$ and this requires to re-express $C(Y, Z)$ in terms of new variables $\bar{Y}$ :

$$
\bar{Y}_{1}:=\partial_{U_{1}} \cdot \partial_{X_{2}}, \quad \bar{Y}_{2}:=-\partial_{U_{2}} \cdot \partial_{X_{1}}, \quad \bar{Y}_{3}:=\frac{1}{2} \partial_{U_{3}} \cdot\left(\partial_{X_{1}}-\partial_{X_{2}}\right),
$$

which are nothing but the integration-by-parts versions of the $Y$-variables (up to divergence terms, which we disregard). Notice as well that we have also redefined $\bar{Y}_{3}$ in order to get more symmetric expressions. As a result, the coupling $C(Y, Z)$ of eq. (2.4) can be written, up to integrations by parts, as a new function $\bar{C}(\bar{Y}, Z)$ in different variables:

$$
C(Y, Z) \stackrel{\mathrm{TT}}{=} \bar{C}(\bar{Y}, Z)=\left.e^{\lambda\left(Z_{1} \partial_{\bar{Y}_{2}}+Z_{2} \partial_{\bar{Y}_{1}}+Z_{1} Z_{2} \partial_{G}\right) \partial_{\bar{Y}_{3}} K(\bar{Y}, G)}\right|_{G=G(\bar{Y}, Z)},
$$

where we have taken into account the contributions coming from the delta function in the measure. The difference between $\bar{C}(Y, Z)$ and $C(Y, Z)$ lies in the operators appearing in the exponent - the former has only three terms while the latter (2.5) has seven terms. In particular, in flat space, they simply coincide with each other.

Given all this, we can express the gauge variation (3.3) as

$$
\delta_{E_{1}}^{(0)} S^{(3)} \stackrel{\mathrm{TT}}{=} \int_{(\mathrm{A}) \mathrm{dS}} e^{\partial_{U_{1}} \cdot \partial_{U_{2}}}\left[T_{12}\left(X, U_{1}\right) \partial_{X}^{2} \Phi_{3}\left(X, U_{2}\right)+T_{13}\left(X, U_{1}\right) \partial_{X}^{2} \Phi_{2}\left(X, U_{2}\right)\right]_{U_{i}=0},
$$


where $T_{12}(X, U)$ is given by

$$
T_{12}(X, U)=\left.\frac{1}{2} \partial_{Y_{1}} \bar{C}(Y, Z) E_{1}\left(X_{1}, U_{1}\right) \Phi_{2}\left(X_{2}, U_{2}\right)\right|_{\substack{X_{i}=X \\ U_{i}=0}},
$$

in terms of the newly defined variables $Y$ and $Z$ (for simplicity of notations):

$$
\begin{array}{lll}
Y_{1}=\partial_{U_{1}} \cdot \partial_{X_{2}}, & Y_{2}=-\partial_{U_{2}} \cdot \partial_{X_{1}}, & Y_{3}=\frac{1}{2} U \cdot\left(\partial_{X_{1}}-\partial_{X_{2}}\right), \\
Z_{1}=U \cdot \partial_{U_{2}}, & Z_{2}=U \cdot \partial_{U_{1}}, & Z_{3}=\partial_{U_{1}} \cdot \partial_{U_{2}} \cdot
\end{array}
$$

Finally, comparing eq. (3.6) with eq. (3.2), we obtain the first-order deformation of the gauge transformations as

$$
\delta_{E_{1}}^{(1)} \Phi_{3}(X, U) \stackrel{\text { TT }}{=}-T_{12}(X, U)+U^{2} \alpha(X, U)+U \cdot X \beta(X, U)+U \cdot \partial_{X} \gamma(X, U) .
$$

Several comments are in order. First, the $\stackrel{\text { TT }}{=}$ above is to be considered with respect to the generating functions $E_{1}$ and $\Phi_{2}$ and it is inherited from the analogous equivalence relation on $\Phi_{1}$ and $\Phi_{2}$ in (3.6); second, $\alpha$ and $\gamma$ terms are in the kernel of the TT projection and so are inherited from the $\Phi_{3}$ part of the equality modulo traces and divergences; lastly, the $\beta$ term is introduced since the field 3 is tangent. Hence, simplifying the problem by restricting our attention to the TT part seems to leave too many ambiguities in the determination of $\delta_{E_{1}}^{(1)} \Phi_{3}$, but actually, as we will see, these ambiguities can be fixed by general consistencies. Let us make this point clear:

- First, the $\alpha$ term is what would be completely fixed if we had worked with the full vertices instead of their TT parts. Hence, in principle, its determination requires the information about the trace and divergent pieces of the interactions. However, without relying on an explicit treatment of such pieces, the corresponding gauge-algebra deformations can be completely determined from the main piece $T_{i j}$ by asking consistency with the traceless conditions on gauge parameters (in the Fronsdal setting).

- The $\beta$ term is a peculiarity of (A)dS couplings. Since any non-linear field theory in (A)dS can be reformulated in the ambient-space formalism, the gauge transformations of such a theory admit expressions as tangent ambient-space tensors. Hence, by requiring that $\delta_{E_{1}}^{(1)} \Phi_{3}$ be tangent, one can fix $\beta$.

- The last term given by $\gamma$ corresponds to the genuine ambiguity related to redefinitions of gauge parameters.

Hence, modulo the genuine ambiguity $\gamma$ which we shall analyze later, the deformation of gauge transformations can be written as

$$
\delta_{E_{1}}^{(1)} \Phi_{3}(X, U) \stackrel{\mathrm{TT}}{=}-\Pi_{\Phi} T_{12}(X, U),
$$

where $\Pi_{\Phi}$ is the projectors which make the corresponding bracket traceless (by choosing a suitable $\alpha$ ) and renders the expression tangent (by choosing a suitable $\beta$ ) for (A)dS cases. Moreover, we also assume that $\Pi_{\Phi}$ introduces in (A)dS a suitable power of $X^{2} / L^{2}$ as a 
factor (which is in fact another ambiguity not listed above) so that the $\delta_{E_{1}}^{(1)} \Phi_{3}$ satisfies the homogeneity condition as well. Our prescription for $\Pi_{\Phi}$ may look a bit formal at this stage, but soon it will become clear that in this way we have access to many interesting informations on HS interactions.

The expression (3.10) for the first-order deformation of gauge transformations is never vanishing for any of the cubic interactions (2.7). However, as anticipated, some of these deformations are trivial since they can be reabsorbed by a gauge-field redefinition.

Redefinitions of gauge fields and parameters. In order to analyze the effects of gauge-field and gauge-parameter redefinitions, let us first express them in our framework. Without loss of generality, we concentrate on redefinitions of $\Phi_{3}$ and $E_{3}$, which are given in terms of functions $\Omega_{3}, \Delta_{12}$ and $\Delta_{21}$ as

$$
\begin{aligned}
\Phi_{3}(X, U) \rightarrow & \Phi_{3}(X, U)+\left.\Pi_{\Phi} \Omega_{3}(A, B, Y, Z) \Phi_{1}\left(X_{1}, U_{1}\right) \Phi_{2}\left(X_{2}, U_{2}\right)\right|_{\substack{X_{i}=X \\
U_{i}=0}}, \\
E_{3}(X, U) \rightarrow & E_{3}(X, U)+\left.\Pi_{E} \Delta_{12}(A, B, Y, Z) E_{1}\left(X_{1}, U_{1}\right) \Phi_{2}\left(X_{2}, U_{2}\right)\right|_{\substack{X_{i}=X \\
U_{i}=0}} \\
& +\left.\Pi_{E} \Delta_{21}(A, B, Y, Z) E_{2}\left(X_{1}, U_{1}\right) \Phi_{1}\left(X_{2}, U_{2}\right)\right|_{\substack{X_{i}=X \\
U_{i}=0}} .
\end{aligned}
$$

Here, we introduced the operators $A$ and $B$ defined by

$$
A=\frac{1}{2} U \cdot \partial_{X}=\frac{1}{2} U \cdot\left(\partial_{X_{1}}+\partial_{X_{2}}\right), \quad B=\partial_{X_{1}} \cdot \partial_{X_{2}} .
$$

As before, we introduce as well the projectors $\Pi_{\Phi}$ and $\Pi_{E}$ in order to make the redefinitions compatible with traceless and tangent gauge parameters. The projector $\Pi_{E}$ has exactly the same role as $\Pi_{\Phi}$, except that it adjusts the degree of homogeneity to match that of gauge parameters.

\subsection{Non-deforming couplings}

We are now ready to examine how the redefinitions (3.11) contribute to the first-order deformations of the gauge-transformations. When taking into account such contributions, $\delta_{E_{1}}^{(1)} \Phi_{3}$ takes the following form:

$$
\delta_{E_{1}}^{(1)} \Phi_{3} \stackrel{\mathrm{TT}}{=} \Pi_{\Phi}\left(-\frac{1}{2} \partial_{Y_{1}} \bar{C}+\left[\Omega_{3}, U_{1} \cdot \partial_{X_{1}}\right]+U \cdot \partial_{X} \Delta_{12}\right) E_{1} \Phi_{2} \underset{\substack{X_{i}=X \\ U_{i}=0}}{ }
$$

where, using the chain rule, the commutator can be written as

$$
\left[\Omega_{3}, U_{1} \cdot \partial_{X_{1}}\right]=\left(A \partial_{Z_{2}}+B \partial_{Y_{1}}+Y_{3} \partial_{Z_{2}}-Y_{2} \partial_{Z_{3}}\right) \Omega_{3}
$$

Hence, for a given cubic interaction $\bar{C}$, the corresponding $\delta_{E_{1}}^{(1)} \Phi_{3}$ vanishes if and only if there exist functions $\Omega_{3}$ and $\Delta_{12}$ such that

$$
\begin{aligned}
-\frac{1}{2} \partial_{Y_{1}} \bar{C}(Y, Z)+\left(A \partial_{Z_{2}}+B \partial_{Y_{1}}+Y_{3} \partial_{Z_{2}}-\right. & \left.Y_{2} \partial_{Z_{3}}\right) \Omega_{3}(A, B, Y, Z) \\
& +2 A \Delta_{12}(A, B, Y, Z)=0 .
\end{aligned}
$$


The $A$-dependence can be trivially solved by taking $\Delta_{12}=-\partial_{Z_{2}} \Omega_{3} / 2$ and assuming $\Omega_{3}$ independent of $A$. Then the equation simplifies into

$$
-\frac{1}{2} \partial_{Y_{1}} \bar{C}(Y, Z)+\left(B \partial_{Y_{1}}+Y_{3} \partial_{Z_{2}}-Y_{2} \partial_{Z_{3}}\right) \Omega_{3}(B, Y, Z)=0 .
$$

In principle, for each cubic-interaction vertex:

$$
\bar{P}_{s_{1} s_{2} s_{3}}^{[n]}(Y, Z):=\left.e^{\lambda\left(Z_{1} \partial_{Y_{2}}+Z_{2} \partial_{Y_{1}}+Z_{1} Z_{2} \partial_{G}\right) \partial_{Y_{3}}} Y_{1}^{s_{1}-n} Y_{2}^{s_{2}-n} Y_{3}^{s_{3}-n} G^{n}\right|_{G=G(Y, Z)},
$$

one can examine whether there exists $\Omega_{3}$ satisfying eq. (3.16) or not.

Flat-space case. In flat space, eq. (3.16) can be written as

$$
\left(B \partial_{Y_{1}}+Y_{3} \partial_{Z_{2}}-Y_{2} \partial_{Z_{3}}\right)[C(Y, Z)+2 B \Omega(B, Y, Z)]=0,
$$

using the fact that $C=\bar{C}$ satisfies $\left(Y_{i} \partial_{Z_{j}}-Y_{j} \partial_{Z_{i}}\right) C=0$. The solution is given as an arbitrary polynomial in six variables:

$$
C+2 B \Omega=\sum_{l_{i}, m_{i}, n_{i}} g_{l_{2}, l_{3}, m_{2}, m_{3}, n_{2}, n_{3}} Y_{2}^{l_{2}} Y_{3}^{l_{3}} h_{12}^{m_{2}} h_{13}^{m_{3}} f_{2}^{n_{2}} f_{3}^{n_{3}},
$$

where the $h_{1 i}$ 's and the $f_{i}$ 's are given by

$$
\begin{array}{ll}
h_{12}(B, Y, Z):=-Y_{1} Y_{2}-B Z_{3}, & f_{2}(B, Y, Z):=Y_{2} G(Y, Z)+B Z_{1} Z_{3}, \\
h_{13}(B, Y, Z):=-Y_{1} Y_{3}+B Z_{2}, & f_{3}(B, Y, Z):=-Y_{3} G(Y, Z)+B Z_{1} Z_{2} .
\end{array}
$$

One can notice that $h_{1 i} \approx H_{1 i}$ up to integrations by parts. Moreover, the $f_{i}$ 's can be also related to $H$-like objects, $h_{23}^{ \pm}:=-Y_{2} Y_{3} \pm B Z_{1}$, as

$$
B f_{2}=Y_{2}^{2} h_{13}+h_{12} h_{23}^{-}, \quad B f_{3}=Y_{3}^{2} h_{12}+h_{13} h_{23}^{+} .
$$

In fact, a subset - but not all — of the couplings (3.19) can be recast into $H$-couplings of the form:

$$
C(Y, Z) \approx \tilde{K}\left(Y_{\ell}, H_{12}, H_{23}, H_{31}\right) \quad[\ell=2 \text { or } 3] .
$$

In order to see this point more clearly, let us restrict our attention to the $s_{1}-s_{2}-s_{3}$ interactions. Then, any consistent coupling is a linear combination of $Y_{1}^{s_{1}-n} Y_{2}^{s_{2}-n} Y_{3}^{s_{3}-n} G^{n}$ with $n=0, \ldots, s_{\min }$. On the other hand, the solution (3.19) tells us that any coupling with $\delta_{E_{1}}^{(1)}=0$ is a linear combination of

$$
Y_{2}^{l_{2}} Y_{3}^{l_{3}}\left(Y_{1} Y_{2}\right)^{m_{2}}\left(Y_{1} Y_{3}\right)^{m_{3}}\left(Y_{2} G\right)^{n_{2}}\left(Y_{3} G\right)^{n_{3}}=Y_{1}^{m_{2}+m_{3}} Y_{2}^{l_{2}+m_{2}+n_{2}} Y_{3}^{l_{3}+m_{3}+n_{3}} G^{n_{2}+n_{3}},
$$

with non-negative integers $l_{i}, m_{i}, n_{i}$ 's satisfying

$$
s_{1}=m_{2}+m_{3}+n_{2}+n_{3}, \quad s_{2}=l_{2}+m_{2}+2 n_{2}+n_{3}, \quad s_{3}=l_{3}+m_{3}+n_{2}+2 n_{3} .
$$

Analyzing the above inequalities, one can conclude that, among all possible values of $n=$ $0, \ldots, s_{\min }$, the non-deforming couplings with $\delta_{E_{1}}^{(1)}=0$ correspond to those satisfying

$$
n \leq \frac{s_{2}+s_{3}-s_{1}}{2} \text {. }
$$


Equivalently, one can conclude that the number of derivatives, $\#_{\partial}$, of such couplings satisfies

$$
\left(\#_{\partial} \text { in a flat-space coupling with } \delta_{E_{1}}^{(1)}=0\right) \geq 2 s_{1} \text {. }
$$

Now, let us move to the $H$-couplings given by eq. (3.22). The analysis carried out in section 6.2 shows that they correspond to the couplings with

$$
n \leq \frac{s_{1}+s_{2}+s_{3}}{2}-\max \left\{s_{1}, s_{\text {mid }}\right\} .
$$

Hence, we have two distinct cases, $s_{1} \neq s_{\min }$ and $s_{1}=s_{\min }$ :

- $s_{\mathbf{1}} \neq s_{\text {min }}$ : the two inequalities (3.25) and (3.27) coincide with each other. Hence, any coupling with $\delta_{E_{1}}^{(1)}=0$ can be written as a $H$-coupling (3.22);

- $s_{1}=s_{\min }:$ in this case, there exist couplings in the range:

$$
\frac{s_{\max }+s_{\min }-s_{\operatorname{mid}}}{2}<n \leq \frac{s_{2}+s_{3}-s_{1}}{2},
$$

which neither deform $\delta_{E_{1}}^{(1)}$ nor are expressible as a $H$-coupling (3.22).

(A)dS case. For the analysis of (A)dS cubic interactions, we need to come back to the original condition (3.16). The basic way of solving the problem is Taylor expanding $\Omega_{3}$ as

$$
\Omega_{3}(B, Y, Z)=\sum_{m=0}^{\infty} B^{m} \Omega^{[m]}(Y, Z),
$$

and recasting (3.16) into a set of equations:

$$
\partial_{Y_{1}} \Omega^{[m]}(Y, Z)=\left(Y_{2} \partial_{Z_{3}}-Y_{3} \partial_{Z_{2}}\right) \Omega^{[m-1]}(Y, Z) .
$$

For a given $\Omega^{[-1]}=\frac{1}{2} \bar{C}(Y, Z)$, we recursively solve for $\Omega^{[m]}$ 's. If there is no obstruction in solving (3.30) to all order, then the corresponding $\bar{C}(Y, Z)$ does not induce any non-trivial deformation of the gauge transformations. For this analysis, let us first consider $\Omega^{[-1]}$ given by a monomial:

$$
\Omega^{[-1]}(Y, Z)=Y_{1}^{\sigma_{1}} Y_{2}^{\sigma_{2}} Y_{3}^{\sigma_{3}} Z_{1}^{\tau_{1}} Z_{2}^{\tau_{2}} Z_{3}^{\tau_{3}} .
$$

Then, one can show that the equations (3.30) admit a solution if (see appendix C):

$$
\sigma_{2}-\tau_{2} \geq \sigma_{1} \quad \text { or } \quad \sigma_{3}-\tau_{3} \geq \sigma_{1} .
$$

However, depending on linear combinations of monomials (3.31), a solution may exist for a wider class of polynomials $\Omega^{[-1]}$, although each monomial term does not admit a solution independently. For instance, for the flat-space couplings, the bound is given in (3.25) which is equivalent to

$$
\sigma_{2}-\tau_{2}+\sigma_{3}-\tau_{3} \geq \sigma_{1}
$$

One can show that this is weaker than (3.32), although it is not manifest. Having this in mind, let us consider the case $\bar{C}=\bar{P}_{s_{1} s_{2} s_{3}}^{[3]}(3.17)$. One can first notice that $\Omega^{[-1]}$ can be 
expanded as a power series in $\lambda$, and examined independently for each of the expansion coefficients. Hence, we can focus on

$$
\Omega^{[-1]}(Y, Z)=\left.\left(Z_{1} \partial_{Y_{2}}+Z_{2} \partial_{Y_{1}}+Z_{1} Z_{2} \partial_{G}\right)^{k} \partial_{Y_{3}}^{k} Y_{1}^{s_{1}-n} Y_{2}^{s_{2}-n} Y_{3}^{s_{3}-n} G^{n}\right|_{G=G(Y, Z)} .
$$

Each term in the above function, as the monomials (3.31), satisfies

$$
\sigma_{1}=s_{1}, \quad \sigma_{2}-\tau_{2}=s_{2}-n-k, \quad \sigma_{3}-\tau_{3}=s_{3}-n-k,
$$

and should satisfy (3.32) in generic cases or a slightly weaker condition as in the case of (3.33). The precise analysis - whether the differential operator in (3.34) generates a particular linear combination which extends the bound (3.32) - is tedious and nontrivial, so let us draw some general lessons. If the flat-space limit of a (A)dS coupling induces a non-trivial deformation, then it also does in (A)dS: this allows us to examine $\bar{C}=\bar{P}_{s_{1} s_{2} s_{3}}^{[n]}$ only for $n \geq\left(s_{2}+s_{3}-s_{1}\right) / 2$ for the identification of non-deforming couplings. However, the inverse is not true in general: even for the couplings whose flat-space limit satisfies (3.33), and hence does not lead to any non-trivial deformation, their $\Lambda^{k}$ corrections corresponding to (3.34) can induce a non-trivial $\delta^{(1)}$ for a large enough $k$. The reason is that the two quantities $\sigma_{2}-\tau_{2}$ and $\sigma_{3}-\tau_{3}$ appearing in the generic bound (3.32) and also in the special bound (3.33) both decrease as $k$ increases. Hence, there must exist a critical value $k=k_{1}^{c}\left(s_{1}, s_{2}, s_{3}, n\right)$, where the equation (3.30) admits no solution, and the deformation of gauge transformation induced by the coupling $\bar{C}=\bar{P}_{s_{1} s_{2} s_{3}}^{[n]}$ reads

$$
\delta_{E_{1}}^{(1)} \Phi_{3}=\mathcal{O}\left(\Lambda^{k_{1}^{c}\left(s_{1}, s_{2}, s_{3}, n\right)}\right) .
$$

Although we do not identify $k_{1}^{c}\left(s_{1}, s_{2}, s_{3}, n\right)$ precisely, we can have some information on it. For instance, following the generic bound (3.32), we can conclude that $k_{1}^{c}$ satisfies

$$
k_{1}^{c}>\max \left\{s_{2}, s_{3}\right\}-s_{1}-n,
$$

and in the particular case of the minimum-derivative $2-s-s$ interactions, it is

$$
k_{1}^{c}=s-2,
$$

by compatibility with the Fradkin-Vasiliev results [20, 21]. The equation (3.36) may give us a wrong impression that in $(\mathrm{A}) \mathrm{dS}$ all couplings induce non-trivial deformations. It is because we have considered the deformations of a single coupling $\bar{C}=\bar{P}_{s_{1} s_{2} s_{3}}^{[n]}$ alone. For the analysis of deformations, in flat space, it was sufficient to examine each monomial $\bar{P}_{s_{1} s_{2} s_{3}}^{[t]}$ since different couplings never talk to each other having different number of derivatives. On the contrary, this is no more true in $(\mathrm{A}) \mathrm{dS}$, so we need to consider general linear combinations with a fixed number of highest derivatives:

$$
\bar{Q}_{s_{1} s_{2} s_{3}}^{[n]}=\bar{P}_{s_{1} s_{2} s_{3}}^{[n]}+c_{1} \lambda \bar{P}_{s_{1} s_{2} s_{3}}^{[n+1]}+c_{2} \lambda^{2} \bar{P}_{s_{1} s_{2} s_{3}}^{[n+2]}+\cdots \quad\left[c_{1}, c_{2}, \ldots \in \mathbb{R}\right] .
$$

Hence, there may exist more than two terms in the above giving the same leading $\Lambda$-order deformations. In such cases, those deformations might be cancelled among each others. In 
that regard, let us note that, as in the flat-space case, one can choose a basis of couplings such that the ones satisfying the condition (3.27) are expressed as $H$-couplings (3.22) and hence do not induce any deformation $\delta_{E_{1}}^{(1)}$. In other words, starting from the $H$-couplings, one can derive the proper linear combinations $\bar{Q}_{s_{1} s_{2} s_{3}}^{[n]}$ (3.39) leading to precise cancellation of $\delta_{E_{1}}^{(1)}$ (for more detailed analysis, see section 6.2). Hence, one can summarize the result as follows:

- $s_{1} \neq s_{\min }$ : for $n \leq \frac{s_{2}+s_{3}-s_{1}}{2}$, one can find the couplings $\bar{Q}_{s_{1} s_{2} s_{3}}^{[n]}$ which are related to $H$-couplings, so they are manifestly all non-deforming;

- $s_{1}=s_{\min }$ : for $n$ satisfying (3.28), the corresponding gauge-transformation deformation is generically non-trivial. However, one needs in principle to examine all possible $\bar{Q}_{s_{1} s_{2} s_{3}}^{[n]}$ 's in order to see whether there might be a further cancellation leading to $\delta_{E_{1}}^{(1)}=0$. Let us note first that when $s_{1} \leq \frac{s_{2}+s_{3}-s_{1}}{2}$, the minimum-derivative coupling with $n=s_{1}$ also belongs to this class, and it is clear for this coupling that the deformation cannot be cancelled since there is no other term in (3.39). More generally, one can conclude that for the latter couplings this cancellation never happens because otherwise it would contradict the compatibility with the non-triviality of the global-symmetry deformations (see section 5).

Remarks. The results obtained in this section are summarized in table 1. Let us conclude with a few more remarks:

- One can see that $\delta_{E_{i}}^{(1)} \Phi_{i+1}=0$ implies $\delta_{E_{i}}^{(1)} \Phi_{i-1}=0$ and vice versa. Hence, when we discuss whether the deformations are vanishing or not, we can refer them to $\delta_{E_{i}}^{(1)}$ without writing explicitly on which field they act upon.

- The $H$-couplings (3.22) are non-deforming since $Y_{2}, Y_{3}$ and $H_{i j}$ 's commute with the free gauge transformation $\delta_{E_{1}}^{(0)} \Phi_{3}$ without relying on the EoM, irrespectively of the curvature of spacetime. However, if we allow the dependence of both $Y_{2}$ and $Y_{3}$ at the same time in the coupling (3.22), then it does not satisfy the (A)dS gauge invariance condition any more, so it is important to allow only one between $Y_{2}$ and $Y_{3}$.

- In flat space, there is a class of couplings with $s_{1}=s_{\min }$ satisfying the condition (3.28) for which only two of the three deformations are non-vanishing:

$$
\delta_{E_{1}}^{(1)}=0, \quad \delta_{E_{2,3}}^{(1)} \neq 0
$$

The corresponding (A)dS couplings deform all three gauge transformations:

$$
\delta_{E_{1}}^{(1)}=\mathcal{O}\left(\Lambda_{1}^{k_{1}^{c}}\right), \quad \delta_{E_{2,3}}^{(1)}=\mathcal{O}\left(\Lambda^{0}\right) \neq 0
$$

due to the presence of lower-derivative terms in the coupling. 
Examples: gravitational interactions of spin-three and -four fields. Let us conclude this section with some concrete examples: the cubic vertices for the $3-3-2$ and 4-4-2 interactions. These are of particular interest since they show how HS fields (spin 3 and spin 4) can gravitationally interact with spin 2 in a (A)dS background. In each cases, there exist three consistent vertices, while only the ones with the minimum number of highest-derivatives (four for spin 3 and six for spin 4):

$$
K^{3-3-2}(Y, G)=\frac{k}{2} Y_{1} Y_{2} G^{2}, \quad K^{4-4-2}(Y, G)=\frac{k^{\prime}}{8} Y_{1}^{2} Y_{2}^{2} G^{2},
$$

induce non-trivial deformations of gauge transformations. Following the analysis presented in this section, one can compute the corresponding $\bar{C}$ 's by applying $e^{\lambda\left(Z_{1} \partial_{Y_{2}}+Z_{2} \partial_{Y_{1}}+Z_{1} Z_{2} \partial_{G}\right) \partial_{Y_{3}}}$ and then extract the gauge-transformation deformation $\delta^{(1)}$ according to (3.13). With proper gauge-parameter redefinitions, controlled by $\Delta_{i j}$ 's, one can remove the higherderivative contributions in $\delta^{(1)}$. All in all, under the spin-2 gauge transformation with parameter $\Xi$, the spin-3 field $\Phi$ transforms as

$$
\begin{aligned}
\delta_{\Xi}^{(1)} \Phi(X, U) \stackrel{\mathrm{TT}}{=}-\left.3 k \lambda\left(\frac{1}{3 !} Y_{1} Z_{1}^{3}+\frac{1}{2} Y_{2} Z_{1}^{2} Z_{2}\right) \Xi\left(X_{1}, U_{1}\right) \Phi\left(X_{2}, U_{2}\right)\right|_{\substack{X_{i}=X \\
U_{i}=0}} \\
=-\left.3 k \lambda\left(\partial_{U_{1}} \cdot \partial_{X_{2}}-\partial_{X_{1}} \cdot \partial_{U_{2}}\right) \Xi\left(X_{1}, U_{1}\right) \Phi\left(X_{2}, U_{2}\right)\right|_{\substack{X_{i}=X \\
U_{i}=U}},
\end{aligned}
$$

and the spin-4 field $\Psi$ as

$$
\begin{aligned}
\delta_{\Xi}^{(1)} \Psi(X, U) \stackrel{\mathrm{TT}}{=}-\left.6 k^{\prime} \lambda^{2}\left(\frac{1}{4 !} Y_{1} Z_{1}^{4}+\frac{1}{3 !} Y_{2} Z_{1}^{3} Z_{2}\right) \Xi\left(X_{1}, U_{1}\right) \Psi\left(X_{2}, U_{2}\right)\right|_{\substack{X_{i}=X \\
U_{i}=0}} \\
=-\left.6 k^{\prime} \lambda^{2}\left(\partial_{U_{1}} \cdot \partial_{X_{2}}-\partial_{X_{1}} \cdot \partial_{U_{2}}\right) \Xi\left(X_{1}, U_{1}\right) \Psi\left(X_{2}, U_{2}\right)\right|_{\substack{X_{i}=X \\
U_{i}=U}} .
\end{aligned}
$$

Analogous expressions can be easily extracted in the general case of $s-s-2$ interactions. As one can notice, these transformations are given by Lie derivatives and correspond to nothing but the general coordinate transformations of tensor fields $\Phi$ and $\Psi$. Hence, one can interpret these interactions as gravitational ones. Notice also that the transformations are proportional to $\lambda \sim \Lambda$ or $\lambda^{2} \sim \Lambda^{2}$, hence they vanish in the flat-space limit. It is also interesting to note that the gravitational interactions (3.42) of HS fields induce deformations also for the graviton field $\Gamma$ : under the spin-3 gauge transformation with parameter $E$, it transforms as

$$
\begin{gathered}
\delta_{E}^{(1)} \Gamma(X, U) \stackrel{\mathrm{TT}}{=}-\left.3 k B Z_{3}\left(\frac{1}{2} Y_{1} Z_{1}^{2}+Y_{2} Z_{1} Z_{2}\right) E\left(X_{1}, U_{1}\right) \Phi\left(X_{2}, U_{2}\right)\right|_{\substack{X_{i}=X \\
U_{i}=0}} \\
=-\left.3 k\left(\partial_{U_{1}} \cdot \partial_{X_{2}}-\partial_{X_{1}} \cdot \partial_{U_{2}}\right) \partial_{X_{1}} \cdot \partial_{X_{2}} \partial_{U_{1}} \cdot \partial_{U_{2}} E\left(X_{1}, U_{1}\right) \Phi\left(X_{2}, U_{2}\right)\right|_{\substack{X_{i}=X \\
U_{i}=U}},
\end{gathered}
$$

while under the spin-4 gauge transformation with parameter $F$, it transforms as

$$
\begin{aligned}
& \delta_{F}^{(1)} \Gamma(X, U) \stackrel{\mathrm{TT}}{=}-\left.\frac{3}{2} k^{\prime} B^{2} Z_{3}^{2}\left(\frac{1}{2} Y_{1} Z_{1}^{2}+Y_{2} Z_{1} Z_{2}\right) F\left(X_{1}, U_{1}\right) \Psi\left(X_{2}, U_{2}\right)\right|_{\substack{X_{i}=X \\
U_{i}=0}} \\
= & -\left.6 k^{\prime}\left(\partial_{U_{1}} \cdot \partial_{X_{2}}-\partial_{X_{1}} \cdot \partial_{U_{2}}\right) \frac{\left(\partial_{X_{1}} \cdot \partial_{X_{2}}\right)^{2}}{2} \frac{\left(\partial_{U_{1}} \cdot \partial_{U_{2}}\right)^{2}}{2} F\left(X_{1}, U_{1}\right) \Psi\left(X_{2}, U_{2}\right)\right|_{\substack{X_{i}=X \\
U_{i}=U}} \cdot(3
\end{aligned}
$$


Such transformations of the spin-2 field do not involve $\lambda$ dependence, they survive in the flat-space limit. Let us also remark that all these expressions (3.43)-(3.46) are tangent and homogeneous with the proper degrees.

\section{Deformations of gauge algebras and abelian couplings}

\subsection{Deformations of gauge algebras}

After obtaining the first order deformation of the gauge transformations $\delta_{E_{i}}^{(1)}$, we can identify the lowest-order part of the gauge algebra. For that, we need to compute $\delta_{E_{1}}^{(0)} \delta_{E_{2}}^{(1)}$ and $\delta_{E_{2}}^{(0)} \delta_{E_{1}}^{(1)}$. We can restrict the attention to the latter since the former can be obtained from the latter by relabeling. This is given by

$$
\left.\delta_{E_{2}}^{(0)} \delta_{E_{1}}^{(1)} \Phi_{3} \stackrel{\mathrm{TT}}{=} \Pi_{\Phi}\left[-\frac{1}{2} \partial_{Y_{1}} \bar{C}+2 A \Delta_{12}, U_{2} \cdot \partial_{X_{2}}\right] E_{1} E_{2}\right|_{\substack{X_{i}=X \\ U_{i}=0}}
$$

where we have included the contribution of gauge-parameter redefinitions, but omitted the contribution of gauge-field redefinitions, since they automatically drops out by taking the antisymmetric part to build the commutator. The commutator of two gauge transformations readily takes the form of a free gauge transformation:

$$
\delta_{\left[E_{2}\right.}^{(0)} \delta_{\left.E_{1}\right]}^{(1)} \Phi_{3}=U \cdot \partial_{X} \llbracket E_{2}, E_{1} \rrbracket_{3}^{(0)},
$$

with the gauge parameter given by

$$
\begin{gathered}
\llbracket E_{1}, E_{2} \rrbracket_{3}^{(0) \stackrel{\mathrm{TT}}{=} \frac{1}{2} \Pi_{E}[}\left[\frac{1}{2}\left(\partial_{Y_{1}} \partial_{Z_{1}}+\partial_{Y_{2}} \partial_{Z_{2}}\right) \bar{C}-\left(A \partial_{Z_{1}}-B \partial_{Y_{2}}-Y_{3} \partial_{Z_{1}}+Y_{1} \partial_{Z_{3}}\right) \Delta_{12}\right. \\
\left.+\left(A \partial_{Z_{2}}+B \partial_{Y_{1}}+Y_{3} \partial_{Z_{2}}-Y_{2} \partial_{Z_{3}}\right) \Delta_{21}\right]\left.E_{1} E_{2}\right|_{\substack{X_{i}=X \\
U_{i}=0}}
\end{gathered}
$$

Here we have used the fact that

$$
\Pi_{\Phi} U \cdot \partial_{X}=U \cdot \partial_{X} \Pi_{E} \cdot
$$

Hence, the formula (4.3) provides the general expression for the lowest-order deformation of gauge algebras (modulo the terms proportional to divergences and d'Alembertians of gauge parameters).

\subsection{Abelian interactions}

We can now ask for a given cubic interaction $\bar{C}$ whether the resulting commutators are Abelian, that is $\llbracket E_{1}, E_{2} \rrbracket_{3}^{(0)}=0$, up to a gauge-parameter redefinition. This is equivalent to ask whether there exists a solution $\left(\Delta_{12}, \Delta_{21}\right)$ to

$$
\begin{aligned}
\frac{1}{2}\left(\partial_{Y_{1}} \partial_{Z_{1}}+\partial_{Y_{2}} \partial_{Z_{2}}\right) \bar{C}(Y, Z) & -\left(A \partial_{Z_{1}}-B \partial_{Y_{2}}-Y_{3} \partial_{Z_{1}}+Y_{1} \partial_{Z_{3}}\right) \Delta_{12}(A, B, Y, Z) \\
& +\left(A \partial_{Z_{2}}+B \partial_{Y_{1}}+Y_{3} \partial_{Z_{2}}-Y_{2} \partial_{Z_{3}}\right) \Delta_{21}(A, B, Y, Z)=0
\end{aligned}
$$

The above equation has a similar structure to the one (3.16) associated with $\delta^{(1)}$, but is more involved including this time two functions $\Delta_{12}$ and $\Delta_{21}$. Hence, we take a different 
approach, instead of solving directly eq. (4.6), ${ }^{7}$ to address the issue of gauge-algebra deformation. For that, let us begin by considering the following proposition: for a given cubic interaction $\bar{C}$, the corresponding deformation of gauge algebra is trivial if and only if at least one of two gauge-transformation deformations $\delta_{E_{1}}^{(1)}$ and $\delta_{E_{2}}^{(1)}$ is trivial:

$$
\llbracket E_{1}, E_{2} \rrbracket_{3}^{(0)}=0 \quad \Longleftrightarrow \quad \delta_{E_{1}}^{(1)}=0 \quad \text { or } \quad \delta_{E_{2}}^{(1)}=0 .
$$

The implication $\Leftarrow$ is manifest: assuming $\delta_{E_{1}}^{(1)}=0$, we get $\delta_{\llbracket E_{1}, E_{2} \rrbracket_{3}^{(0)}}^{(0)}=\delta_{E_{1}}^{(0)} \delta_{E_{2}}^{(1)}$ which is precisely the form of a gauge-parameter redefinition. The implication $\Rightarrow$ can be shown by checking that the couplings with non-trivial global-symmetry deformations - which necessarily deform the gauge algebra - exhaust all couplings with $\delta_{E_{1}}^{(1)}, \delta_{E_{2}}^{(1)} \neq 0$. Leaving the issue of the global-symmetry deformations to the next section, let us conclude this section by presenting the classification separately for flat-space and (A)dS cases.

Flat-space case. Taking the union of the two conditions (3.25), one concludes that a coupling with the number of derivatives:

$$
\#_{\partial}<2 \min \left\{s_{1}, s_{2}\right\}
$$

leads to the deformation of algebra, $\llbracket E_{1}, E_{2} \rrbracket_{3}^{(0)} \neq 0$.

(A)dS case. In (A)dS, only $H$-couplings (3.22) result in a trivial deformation of the gauge transformations. Hence, any coupling with $\llbracket E_{1}, E_{2} \rrbracket_{3}^{(0)}=0$ can be expressed as

$$
C(Y, Z) \approx \tilde{K}\left(Y_{\ell}, H_{12}, H_{23}, H_{31}\right) \quad[\ell=1,2 \text { or } 3]
$$

which corresponds to the union between the $H$-couplings (3.22) and their $\delta_{E_{2}}^{(1)}$ counterparts. This is equivalent to say that a coupling with the number of derivatives:

$$
\# \partial<2 s_{\text {mid }}
$$

leads to a deformation of the algebra, $\llbracket E_{1}, E_{2} \rrbracket_{3}^{(0)} \neq 0$.

\section{Global symmetries}

\subsection{Killing tensors}

The settings for the analysis of gauge-symmetry deformations can be also used to study the global (or rigid) symmetries of HS theory - the HS algebras. The latter can be obtained from the gauge symmetries by imposing the Killing equation: ${ }^{8}$

$$
U \cdot \partial_{X} \bar{E}(X, U)=0 .
$$

\footnotetext{
${ }^{7}$ With the help of Mathematica, we have analyzed all the cases up to spin 10 checking the agreement with the classification obtained in the other way.

${ }^{8}$ See $[51,52]$ for previous works on HS killing tensors.
} 
Together with the traceless condition on the gauge parameter and the homogeneity and tangentiality conditions (1.8), ${ }^{9}$ the Killing equation (5.1) results in additional conditions:

$$
\partial_{X} \cdot \partial_{U} \bar{E}(X, U)=0, \quad \partial_{X}^{2} \bar{E}(X, U)=0, \quad \partial_{U}^{2} \bar{E}(X, U)=0 .
$$

The solutions to these conditions for the parameters $\bar{E}$ — namely Killing tensors — forms a HS algebra. A generic element of the latter:

$$
\bar{E}(X, U)=\sum_{r=0}^{\infty} \frac{1}{(r !)^{2}} \bar{E}_{M[r]} T^{M[r]},
$$

can be decomposed into the generators of the corresponding HS algebra as

$$
T^{M_{1} \cdots M_{r} M_{1}^{\prime} \cdots M_{r}^{\prime}}=X^{\left[M_{1}\right.} U^{\left.M_{1}^{\prime}\right]} \cdots X^{\left[M_{r}\right.} U^{\left.M_{r}^{\prime}\right]} .
$$

In eq. (5.6), we have used a short-hand notation $M[r]$ for the superscript $M_{1} \cdots M_{r} M_{1}^{\prime} \cdots M_{r}^{\prime}$. Let us notice here that since the generators $T^{M[r]}$ appear always contracted with the traceless parameter $\bar{E}_{M[r]}$, they are defined actually as an equivalence class:

$$
T^{M[r]} \sim T^{M[r]}+X \cdot U P_{1}^{M[r]}+X^{2} P_{2}^{M[r]}+U^{2} P_{3}^{M[r]}
$$

where $P_{i}^{M[r]}$ s are arbitrary polynomial in $X$ and $U$ possibly involving the ambient metric tensor $\eta_{M N}$ and compatible with the homogeneity degree of $T^{M[r]}$. From the gauge-algebra brackets (4.3) given by $s_{1}-s_{2}-s_{3}$ cubic interactions, we can then deduce the $r_{1}-r_{2}-r_{3}$ part $\left(r_{i} \equiv s_{i}-1\right)$ of the structure constant.

\subsection{Higher-spin algebras}

Now let us consider the bracket (4.3) where the gauge parameters are Killing tensors. The cubic consistency ensures that the bracket of two Killing tensors gives again a Killing tensor:

$$
U \cdot \partial_{X} \llbracket \bar{E}_{1}, \bar{E}_{2} \rrbracket_{3}^{(0)}=0 .
$$

About the explicit form of the bracket, we have two major simplifications. First, the TT restriction does not exclude any term since all TT parts vanish when the Killing equations

${ }^{9}$ The operators $e=X \cdot \partial_{U}, f=U \cdot \partial_{X}$ and $h=X \cdot \partial_{X}-U \cdot \partial_{U}$ can be viewed as the generators of $\mathfrak{s p}_{2}$ :

$$
[h, e]=2 e, \quad[h, f]=-2 f, \quad[e, f]=h .
$$

Then, the massless Killing tensors are $\mathfrak{s p}_{2}$ singlets reflecting the construction of HS algebra based on Howe duality. For the partially-massless cases with $\mu \in \mathbb{N}$, the Killing tensors carry rather $(2 \mu+1)$-dimensional representations:

$$
e \bar{E}=0, \quad f^{\mu+1} \bar{E}=0, \quad(h-2 \mu) \bar{E}=0 .
$$

On the other hand, the fields carry infinite dimensional representations of $\mathfrak{s p}_{2}$ :

$$
e \Phi=0, \quad(h-\mu) \Phi=0, \quad \Phi \sim \Phi+f^{\mu+1} E \quad[e E=0, \quad(h-2 \mu) E=0] .
$$


hold. Second, the reparameterization terms given by $\Delta_{i j}$ also vanish since they involve $U_{i} \cdot \partial_{X_{i}} \bar{E}_{i}$. Hence, the bracket of the global-symmetry algebra:

$$
\llbracket \bar{E}_{1}, \bar{E}_{2} \rrbracket_{3}^{(0)}=\left.\Pi_{E} \mathcal{F}^{s_{3}} s_{1} s_{2}(Y, Z) \bar{E}_{1} \bar{E}_{2}\right|_{\substack{X_{i}=X \\ U_{i}=0}}
$$

is given simply by

$$
\mathcal{F}^{s_{3}} s_{1} s_{2}(Y, Z)=\frac{1}{4}\left(\partial_{Y_{1}} \partial_{Z_{1}}+\partial_{Y_{2}} \partial_{Z_{2}}\right) \bar{C}(Y, Z)
$$

and there remains no term unfixed or ambiguous. Herefrom, we suppress the superscript (0) of the bracket since it is the complete one for the global symmetries. Incidentally, the absence of ambiguity at the global-symmetry level is also true for $\delta_{\bar{E}_{i}}^{(1)}$. Let us recall that $\bar{C}(Y, Z)$ is given explicitly through the coupling function $K$ which can be expanded for given spins $s_{1}-s_{2}-s_{3}$ as follows:

$$
K\left(Y_{1}, Y_{2}, Y_{3}, G\right)=\sum_{n=0}^{s_{\min }} k_{n} \frac{Y_{1}^{s_{1}-n}}{\left(s_{1}-n\right) !} \frac{Y_{2}^{s_{2}-n}}{\left(s_{2}-n\right) !} \frac{Y_{3}^{s_{3}-n}}{\left(s_{3}-n\right) !} \frac{G^{n}}{n !} .
$$

In the following, we explore the implications of HS global symmetries separately for (A)dS and for flat space.

(A)dS. The coupling $\bar{C}$ contains $K(Y, G)$ as its highest-derivative part, while the lowerderivative parts are generated by the operator $e^{\lambda\left(Z_{1} \partial_{Y_{2}}+Z_{2} \partial_{Y_{1}}+Z_{1} Z_{2} \partial_{G}\right) \partial_{Y_{3}}}$. One can count the number of derivatives involved in the coupling associated with $G^{n}$, and its minimum is given by

$$
s_{1}+s_{2}+s_{3}-2 n-2\left(s_{3}-n\right)=s_{1}+s_{2}-s_{3},
$$

independently on the initial number of derivatives of the coupling. This implies that the lowest-derivative part of the above commutator contains exactly the right number of derivatives to be compatible with the homogeneity conditions (1.8):

$$
\begin{aligned}
\left(\Delta_{X} \text { of } \llbracket E_{1}, E_{2} \rrbracket_{3}\right) & =\left(\Delta_{X} \text { of } E_{1}\right)+\left(\Delta_{X} \text { of } E_{2}\right)-\left(\# \partial \text { of } \llbracket E_{1}, E_{2} \rrbracket_{3}\right) \\
& =\left(s_{1}-1\right)+\left(s_{2}-1\right)-\left(s_{1}+s_{2}-s_{3}-1\right) \\
& =s_{3}-1=\left(\Delta_{X} \text { of } E_{3}\right) .
\end{aligned}
$$

The higher-derivative terms have lower degree of homogeneity in $X$ and need to be supplemented by appropriate powers of $X^{2}$ accordingly to the projector $\Pi_{E}$ - recall that the projector $\Pi_{E}$ is making the commutator traceless, tangent and also of the proper homogeneity degree. Therefore, all the higher-derivative terms are trace components, which are annihilated by the action of $\Pi_{E}$. Hence, the lowest-derivative part of the cubic interaction $\bar{C}$ contains the full information on the structure constant of the global symmetries. The commutator can then be readily computed starting from the explicit formulas for the 
coupling $\bar{C}(Y, Z)$ and reads ${ }^{10}$

$$
\begin{aligned}
& \mathcal{F}_{s_{1} s_{2}}^{s_{3}}\left(Y_{1}, Y_{2}, Z_{1}, Z_{2}, Y_{3} Z_{3}\right)=\frac{1}{4}\left(\partial_{Y_{1}} \partial_{Z_{1}}+\partial_{Y_{2}} \partial_{Z_{2}}\right) \sum_{n=0}^{s_{\min }} k_{n} \lambda^{s_{3}-n} \times \\
& \times\left[\frac{\left(Z_{1} \partial_{Y_{2}}+Z_{2} \partial_{Y_{1}}+Z_{1} Z_{2} \partial_{G}\right)^{s_{3}-n}}{\left(s_{3}-n\right) !} \frac{Y_{1}^{s_{1}-n}}{\left(s_{1}-n\right) !} \frac{Y_{2}^{s_{2}-n}}{\left(s_{2}-n\right) !} \frac{G^{n}}{n !}\right]_{G=G(Y, Z)} .
\end{aligned}
$$

Since the above expression is homogeneous in the radial coordinate, one can also replace the powers of $\lambda$ by

$$
\lambda^{s_{3}-n} \quad \rightarrow \quad(d-5)(d-3) \cdots\left(d+2\left(s_{3}-n\right)-7\right) \Lambda^{s_{3}-n} .
$$

However, in the following we shall keep $\lambda$ for the brevity of expression, unless the above replacement is instructive.

In order to see more transparently the bracket structure, it is convenient to change our convention. First, we remove $Z_{1}$ and $Z_{2}$, by choosing a convention with the evaluation $U_{i}=U$ instead of $U_{i}=0$ :

$$
\llbracket \bar{E}_{1}, \bar{E}_{2} \rrbracket_{3}=\Pi_{E} \mathfrak{F}^{s_{3}} s_{1} s_{2}\left(Y_{1}, Y_{2}, Y_{3} Z_{3}\right) \bar{E}_{1} \bar{E}_{2} \underset{\substack{x_{i}=X \\ U_{i}=U}}{ },
$$

where the new bracket function $\mathfrak{F}^{s_{3}}{ }_{s_{1} s_{2}}$ is related to the old one $\mathcal{F}^{s_{3}}{ }_{s_{1} s_{2}}$ by

$$
\mathfrak{F}^{s_{3}} s_{1} s_{2}\left(Y_{1}, Y_{2}, Y_{3} Z_{3}\right)=\left.\frac{1}{\left(1-\partial_{Z_{1}}\right)\left(1-\partial_{Z_{2}}\right)} \mathcal{F}^{s_{3}}{ }_{s_{1} s_{2}}\left(Y_{1}, Y_{2}, Z_{1}, Z_{2}, Y_{3} Z_{3}\right)\right|_{Z_{1}=0=Z_{2}} .
$$

Second, using the Killing equation, we remove $Y_{3}$ 's at the price of introducing $B$-dependence:

$$
\mathfrak{F}^{s_{3}{ }_{s_{1} s_{2}}}\left(Y_{1}, Y_{2}, B Z_{3}\right)=\left.e^{\frac{1}{2}\left[\left(Y_{1}+Y_{2}\right) \partial_{Z_{3}}+B\left(\partial_{Y_{1}}+\partial_{Y_{2}}\right)\right] Z_{3} \partial_{w}} \mathfrak{F}^{s_{3}}{ }_{s_{1} s_{2}}\left(Y_{1}, Y_{2}, w\right)\right|_{w=0} .
$$

Note that since $Y_{3}$ appears always as $Y_{3} Z_{3}$ in the expression (5.15), $B$ also appears as $B Z_{3}$. As we have mentioned before, any cubic interaction leads to consistent (in the sense of closure) bracket for HS algebra, and one can show that any consistent bracket should have the form:

$$
\mathfrak{F}^{s_{3}}{ }_{s_{1} s_{2}}\left(Y_{1}, Y_{2}, B Z_{3}\right)=\mathfrak{F}_{s_{1} s_{2}}^{s_{3}}\left(G_{3}, H_{3}\right) \quad\left[G_{3}=Y_{1}+Y_{2}, H_{3}=Y_{1} Y_{2}+B Z_{3}\right] .
$$

For concreteness sake, we provide several examples of such brackets obtained from cubic interactions given by coupling constant $k_{n}$ 's (5.12) in table 2 (here we disregard the higherderivative couplings associated with lower $n$ 's in (5.12) which give redundant structures).

At this stage, let us come back to the issue of classifying all non-Abelian vertices. The point is that the map from the space of consistent cubic interactions to the space of consistent brackets:

$$
\{K(Y, G)\} \quad \longrightarrow \quad\left\{\mathfrak{F}^{s_{i}} s_{i+1} s_{i-1}\left(G_{i}, H_{i}\right)\right\}
$$

\footnotetext{
${ }^{10}$ To be more precise, $k_{n}$ 's must involve totally antisymmetric structure constant as well if $s_{1}+s_{2}+s_{3}$ is an odd integer.
} 


\begin{tabular}{|c|c|}
\hline$s_{1}-s_{2}-s_{3}$ & Structure constants \\
\hline $2-3-3$ & $\mathfrak{F}^{3}{ }_{23}=k_{2} 3 \lambda G_{3}, \quad \mathfrak{F}^{2}{ }_{33}=k_{2} G_{3} H_{3}$ \\
\hline $3-3-3$ & $\mathfrak{F}^{3}{ }_{33}=k_{2} \frac{1}{2} \lambda\left(9 G_{3}^{2}-4 H_{3}\right)+k_{3} \frac{1}{2}\left(3 G_{3}^{2}-2 H_{3}\right)$ \\
\hline $3-4-4$ & $\mathfrak{F}^{4}{ }_{34}=k_{2} 3 \lambda^{2}\left(3 G_{3}^{2}-H_{3}\right)+k_{3} 3 \lambda\left(2 G_{3}^{2}-H_{3}\right)$, \\
& $\mathfrak{F}^{3}{ }_{44}=k_{2} \frac{1}{4} \lambda\left(9 G_{3}^{2} H_{3}-5 H_{3}^{2}\right)+k_{3} \frac{1}{2}\left(3 G_{3}^{2} H_{3}-2 H_{3}^{2}\right)$ \\
\hline $4-4-4$ & $\mathfrak{F}^{4}{ }_{44}=k_{3} \lambda\left(8 G_{3}^{3}-9 G_{3} H_{3}\right)+k_{4}\left(2 G_{3}^{3}-3 G_{3} H_{3}\right)$ \\
\hline $3-4-5$ & $\mathfrak{F}^{3}{ }_{45}=k_{3} \frac{1}{2} G_{3} H_{3}^{2}, \quad \mathfrak{F}^{4}{ }_{53}=k_{3} 4 \lambda G_{3} H_{3}, \quad \mathfrak{F}^{5}{ }_{34}=k_{3} 12 \lambda^{2} G_{3}$ \\
\hline
\end{tabular}

Table 2. AdS rigid structure constants induced by various couplings.

is surjective, as one can evince from the above examples. Although we do not provide an explicit demonstration of this point, it should be clear if one reflects the Fradkin-Vasiliev construction in the frame-like approach [20,21, 23, 41]. Hence, one can conclude that, defining $N_{3}$ and $\bar{N}_{3}$ as the numbers of couplings with $\llbracket E_{1}, E_{2} \rrbracket \neq 0$ and $\llbracket \bar{E}_{1}, \bar{E}_{2} \rrbracket \neq 0$ respectively, then

$$
\bar{N}_{3} \leq N_{3}<s_{\text {mid }}-\frac{1}{2}\left(s_{\text {max }}+s_{\text {mid }}-s_{\text {min }}\right),
$$

where the last inequality is obtained counting the number of couplings which can be written as $H$-couplings. Now, let us count the number $\bar{N}_{3}$ of independent structures in the bracket:

$$
\mathfrak{F}_{s_{1} s_{2}}^{s_{3}}\left(G_{3}, H_{3}\right)=\sum_{v, h} c_{v, h} G_{3}^{v} H_{3}^{h} .
$$

Consistency of the number of contractions with the powers of $X$ and $U_{i}$ gives (for more details, see appendix D)

$$
v+2 h=s_{1}+s_{2}-s_{3}-1, \quad v+h \leq \min \left\{s_{1}, s_{2}\right\}-1,
$$

leading to the following bound for $h$ :

$$
\max \left\{s_{1}, s_{2}\right\}-s_{3} \leq h \leq \frac{1}{2}\left(s_{1}+s_{2}-s_{3}-1\right),
$$

so the number of integer $h$ 's corresponds to the number of independent structures:

$$
\bar{N}_{3}=\left\lceil\frac{1}{2}\left(s_{\min }+s_{\text {mid }}-s_{\max }\right)\right\rceil,
$$

where $\lceil x\rceil=\min \{n \in \mathbb{Z} \mid n \geq x\}$. Finally, from the inequality (5.22), one can conclude that $N_{3}=\bar{N}_{3}$.

Finally let us consider the following bilinear form for the Killing tensors:

$$
\left\langle\bar{E}_{1} \mid \bar{E}_{2}\right\rangle=\left.b_{s_{1}} \frac{\left(\partial_{U_{1}} \cdot \partial_{U_{2}}\right)^{r_{1}}}{r_{1} !} \frac{\left(\partial_{X_{1}} \cdot \partial_{X_{2}}\right)^{r_{1}}}{r_{1} !} \bar{E}_{1}\left(X_{1}, U_{1}\right) \bar{E}_{2}\left(X_{2}, U_{2}\right)\right|_{\substack{X_{i}=0 \\ U_{i}=0}}
$$

given by a series of coefficients $b_{s}$ 's. This bilinear form become invariant if it makes the structure constant cyclic:

$$
\left\langle\bar{E}_{3} \mid \llbracket \bar{E}_{1}, \bar{E}_{2} \rrbracket\right\rangle=\left\langle\bar{E}_{1} \mid \llbracket \bar{E}_{2}, \bar{E}_{3} \rrbracket\right\rangle=\left\langle\bar{E}_{2} \mid \llbracket \bar{E}_{3}, \bar{E}_{1} \rrbracket\right\rangle .
$$


The bracket structures $\mathcal{F}^{s_{i}} s_{s_{i+1} s_{i-1}}$ resulted from cubic interactions satisfy this cyclicity condition if $b_{s}$ 's satisfy

$$
\frac{b_{s}}{b_{s-1}}=(d+2 s-7) \Lambda
$$

where we have used the replacement (5.16) to get the above relation. Note that this condition is independent from the choice of coupling constants $k_{n}$, hence all the (A)dS cubic interactions are automatically consistent with the condition (5.28). It is convenient to present the solution of the above equation as

$$
b_{s}=g^{-1}(d-5)(d-3) \cdots(d+2 s-7) \Lambda^{s}=\frac{\lambda^{s}}{g} .
$$

Then, one can rescale the fields so that the invariant form become proportional to the identity: $b_{s}=1$ - this would correspond to the canonical normalization for HS fields. As a result, the structure constants become automatically cyclic, while the kinetic term takes the form:

$$
S_{\text {can. }}^{(2)}[\Phi] \stackrel{\mathrm{TT}}{=}-\left.\frac{1}{2 g} \int_{(\mathrm{A}) \mathrm{dS}} e^{\lambda \partial_{U_{1}} \cdot \partial_{U_{2}}} \Phi\left(X, U_{1}\right) \partial_{X}^{2} \Phi\left(X, U_{2}\right)\right|_{U_{i}=0},
$$

with an explicit dependence on $\lambda$ for each different spin. An important point to remind here is that the rescaling involves the cosmological constant, so that the invariant form is degenerate in the flat-space limit.

The explicit analysis of the brackets provides a one-to-one correspondence between non-Abelian interacting vertices in the metric-like and in the frame-like formalisms. The next step towards the systematics of HS interactions requires the study of the full quartic consistency, which implies in particular that the consistent brackets must satisfy the Jacobi identity. Hence, one may first explore all possible consistent HS algebras (see [53] for the analysis of the algebras involving only symmetric fields, and [54, 55] for their multi-particle extensions). To conclude the discussion of (A)dS global symmetries, let us consider the gauge-algebra deformation induced by the cubic interaction associated with the coupling function:

$$
K(Y, G)=\frac{1}{g} e^{Y_{1}+Y_{2}+Y_{3}},
$$

which does not involve any $G$ dependence, and generates only the highest derivative couplings for each $s_{1}-s_{2}-s_{3}$. The corresponding cubic interaction takes the form:

$$
C=e^{\lambda \mathcal{D}} K=\frac{1}{g} e^{Y_{1}+Y_{2}+Y_{3}+\lambda\left(Z_{1}+Z_{2}+Z_{3}\right)},
$$

which resembles the cubic interaction for the excitations of the first Regge trajectory of the open bosonic string (for simplicity we do not explicitly write down Chan-Paton factors):

$$
C \sim \frac{1}{g_{o}} e^{Y_{1}+Y_{2}+Y_{3}+\frac{1}{\alpha^{\prime}}\left(Z_{1}+Z_{2}+Z_{3}\right)} .
$$

Interestingly, the gauge-algebra deformation induced by (5.33) gives exactly the Moyal bracket:

$$
\llbracket \bar{E}_{1}^{a}, \bar{E}_{2}^{b} \rrbracket^{c}(X, U)=\left.\Pi_{E}\left(d_{a b}^{c} \sinh G_{3}+f_{a b}^{c} \cosh G_{3}\right) \bar{E}_{1}^{a}\left(X_{1}, U_{1}\right) \bar{E}_{2}^{b}\left(X_{2}, U_{2}\right)\right|_{\substack{X_{i}=X \\ U_{i}=U}},
$$




\begin{tabular}{|c|c|}
\hline$s_{1}-s_{2}-s_{3}$ & Structure constants \\
\hline $2-3-3$ & $\mathfrak{F}^{3}{ }_{23}=0, \quad \mathfrak{F}^{2}{ }_{33}=k_{2} G_{3} H_{3}$ \\
\hline $3-3-3$ & $\mathfrak{F}^{3}{ }_{33}=-\frac{3}{2}\left(k_{2}+a\right) B G_{3}^{2}+a B H_{3}+\frac{1}{2} k_{3}\left(3 G_{3}^{2}-2 H_{3}\right)$ \\
\hline $3-4-4$ & $\mathfrak{F}^{4}{ }_{34}=-\left(k_{3}+4 a\right) B H_{3}+6 a B G_{3}^{2}$, \\
& $\mathfrak{F}_{44}^{3}=-\frac{1}{4}\left(k_{2}+16 b\right) B H_{3}^{2}+6 b B G_{3}^{2} H_{3}+\frac{1}{2} k_{3}\left(3 G_{3}^{2} H_{3}-2 H_{3}^{2}\right)$ \\
\hline $4-4-4$ & $\mathfrak{F}_{44}^{4}=2\left(k_{3}+4 a\right) B G_{3}^{3}-6\left(k_{3}+2 a\right) B G_{3} H_{3}+k_{4}\left(2 G_{3}^{3}-3 G_{3} H_{3}\right)$ \\
\hline $3-4-5$ & $\mathfrak{F}^{3}{ }_{45}=\frac{1}{2} k_{3} G_{3} H_{3}^{2}, \quad \mathfrak{F}^{4}{ }_{53}=0, \quad \mathfrak{F}^{5}{ }_{34}=0$ \\
\hline
\end{tabular}

Table 3. Flat rigid structure constants induced by various couplings.

where we have reinstated Chan-Paton factors and the corresponding internal structure constants - totally symmetric $d_{a b c}$ and totally antisymmetric $f_{a b c}$. If the Killing tensors were traceful tensors (so that we do not insert the projector $\Pi_{E}$ ), then the latter would be a good bracket that does even satisfy the Jacobi identity. Notice however that in a setting where finitely many irreducible fields are present for given spin (as in the Vasiliev theory), one should consider only traceless tensors, so that the coupling (5.33) does not actually fulfil the Jacobi identity on traceless tensors. Nevertheless, it is still challenging to reconsider this type of coupling in a HS theory with reducible spectrum [56-62]. Actually, such a reducible theory is a natural candidate for the tensionless limit of the first Reggetrajectory of the open bosonic string.

Flat space. Let us consider now the case of flat-space interactions where the bracket is given by

$$
\mathcal{F}^{s_{3}}{ }_{s_{1} s_{2}}=\frac{1}{4}\left(\partial_{Y_{1}} \partial_{Z_{1}}+\partial_{Y_{2}} \partial_{Z_{2}}\right) \sum_{n=0}^{s_{\min }} k_{n} \frac{Y_{1}^{s_{1}-n}}{\left(s_{1}-n\right) !} \frac{Y_{2}^{s_{2}-n}}{\left(s_{2}-n\right) !} \frac{Y_{3}^{s_{3}-n}}{\left(s_{3}-n\right) !} \frac{[G(Y, Z)]^{n}}{n !} .
$$

One can again remove $Z_{1}, Z_{2}$ by (5.18) and replace the $Y_{3}$-dependence by $B$. However, at this time, $Y_{3}$ and $B$ can appear alone (not always in the combinations $Y_{3} Z_{3}$ and $B Z_{3}$, respectively). The general form of the consistent bracket structure can be derived also for the flat-space case, and it reads

$$
\mathfrak{F}^{s_{3}}{ }_{s_{1} s_{2}}\left(Y_{1}, Y_{2}, B Z_{3}, B\right)=\mathfrak{F}^{s_{3}}{ }_{s_{1} s_{2}}\left(G_{3}, H_{3}, B\right) .
$$

One can notice that, compared to the (A)dS case (5.20), the flat-space brackets may also depend on $B$ in addition to $G_{3}$ and $H_{3}$. We provide some examples for more concrete understanding in table 3 .

As one can see from the appearance of arbitrary coefficients $a$ and $b$, there exist in fact certain functions $\mathfrak{F}^{s_{3}}{ }_{s_{1} s_{2}}\left(G_{3}, H_{3}, B\right)$ which identically vanish when acted on the Killing tensors. Hence, the description (5.37) of the flat-space bracket may involve some redundancies. Even after removing such redundancies (by fixing $a$ and $b$ ), there exist in general more bracket structures than non-Abelian couplings. To iterate, let us remind the reader that any functions of $G_{3}, H_{3}$ and $B$ provide consistent brackets in the sense that the bracket of two Killing tensors gives again a Killing tensor. However, not all such brackets can be associated with consistent cubic interactions: look, for instance, the $3-3-3$ and $4-4-4$ 
examples, which do not involve the structures $G_{3}^{2}$ and $G_{3}^{3}$ respectively. In other words, not all such brackets can be gauged to give consistent cubic interactions in flat space, as opposed to the $(\mathrm{A}) \mathrm{dS}$ case.

Notice that in some cases such as $\mathfrak{F}^{3}{ }_{23}, \mathfrak{F}^{4}{ }_{53}$ and $\mathfrak{F}^{5}{ }_{34}$, there does not exist any nontrivial bracket structure at all. This is one of the generic features of flat-space global symmetries associated with the couplings in Class III in table 1: only one among the three structure constants is non-vanishing. This implies that the invariant bilinear form is necessarily degenerate if one of such couplings is present. In this perspective, of particular interest would be the non-Abelian $s_{1}-s_{2}-2$ interactions with $s_{1}, s_{2}>2$. They have $s_{1}+$ $s_{2}-2$ derivatives and do fall within Class III. Therefore, a consequence of our analysis is that HS generators do not rotate under isometry transformations:

$$
\llbracket T_{s}, T_{2} \rrbracket=0 \quad[\forall s>2],
$$

where $T_{s}$ stands for a Killing tensor associated with spin- $s$ field. This implies again that Gravity is not compatible with HS fields (recall that we have already shown that HS do not transform accordingly to general covariance). Despite the lack of general covariance, one can still study a possible consistency of spin-two non-Abelian (non-gravitational) couplings with HS fields. Schematically, they induce the following brackets: ${ }^{11}$

$$
\llbracket T_{s}, T_{s^{\prime}} \rrbracket \sim T_{2}+\sum_{s^{\prime \prime}>2} T_{s^{\prime \prime}}, \quad \llbracket T_{s}, T_{2} \rrbracket=0 \quad\left[\forall s, s^{\prime}>2\right],
$$

where the terms $\sum_{s^{\prime \prime}>2} T_{s^{\prime \prime}}$ correspond to possible contributions induced by $s_{1}-s_{2}-s_{3}$ interactions with all $s_{i}>2$. Since global symmetries must satisfy the Jacobi identity, the above leads on the one hand to

$$
\llbracket \llbracket T_{s}, T_{s^{\prime}} \rrbracket, T_{2} \rrbracket=-\llbracket \llbracket T_{s^{\prime}}, T_{2} \rrbracket, T_{s} \rrbracket-\llbracket \llbracket T_{2}, T_{s} \rrbracket, T_{s^{\prime}} \rrbracket=0,
$$

while on the other hand to

$$
\llbracket \llbracket T_{s}, T_{s^{\prime}} \rrbracket, T_{2} \rrbracket \sim \llbracket T_{2}+\sum_{s^{\prime \prime}>2} T_{s^{\prime \prime}}, T_{2} \rrbracket \sim \llbracket T_{2}, T_{2} \rrbracket .
$$

Combining the above two conditions, one arrives to the conclusion:

$$
\llbracket T_{2}, T_{2} \rrbracket=0 .
$$

Therefore, one can see that the non-Abelian $s_{1}-s_{2}-2$ couplings with $s_{i}>2$ cannot pass the Jacobi consistency test in a theory whose spin-two charges form the isometry algebra $\llbracket T_{2}, T_{2} \rrbracket \sim T_{2} .{ }^{12}$ In particular, this implies that if a spin-two field couples to HS (even

\footnotetext{
${ }^{11}$ In fact, $\llbracket T_{s}, T_{2} \rrbracket$ can be non-vanishing for $s=3$ if the theory involves the minimum derivative $2-2-3$ coupling. In such a case, the analysis of the Jacobi identity becomes more involved (see appendix E for the details) but the conclusions we draw here do not change anyway.

${ }^{12} \mathrm{~A}$ similar argument can be applied to certain $s_{1}-s_{2}-s$ non-Abelian interactions: the condition that all non-Abelian coupling belong to Class III reads $s_{1}+s_{2} \geq 3 s$. One can also see that when $s=2$, any HS $s_{1}, s_{2}>2$ satisfies this condition.
} 
non-gravitationally but through a non-Abelian coupling), it cannot couple gravitationally to any other field. ${ }^{13}$ If one insists on keeping non-Abelian spin-two self-interactions into the game, then the only allowed $s_{1}-s_{2}-2$ interactions with $s_{i}>2$ are the Abelian ones. In such a theory, non-trivial structure constants may still arise among HS particles, but the global symmetry becomes just a direct sum between the isometry algebra and the algebra generated by HS killing tensors. Hence, the latter can be better interpreted as an internal symmetry. This analysis can be viewed as a field-theoretical version of the ColemanMandula theorem [63]. However, let us emphasize that our conclusions rely neither on the finiteness of the spectrum below a given mass-scale, nor on the locality of the Lagrangian. ${ }^{14}$ Let us note also that we assume the free action to have the standard (Fronsdal) kinetic term: diagonal in fields and of two-derivatives. This assumption forbids to obtain, for instance, the conformal theory of HS $[64,65]$ which actually exists in flat-space background - the spin $s$ part of its kinetic term has $2 s$ derivatives. ${ }^{15}$ It is also worth to comment on the other no-go theorems based on the S-matrix consistency and/or on the general covariance of HS (namely the equivalence principle) [67, 68]. Such results forbid gravitational HS interactions in flat space. Let us mention however that our analysis does not yet rule out the possibility that a non-Abelian self-interacting spin-two interacts with HS fields through Abelian couplings.

\section{Abelian and non-deforming interactions as $\boldsymbol{H}$-couplings}

In the previous section, we have seen that while in flat space the question of gauge-symmetry deformation is equivalent to a simple counting of derivatives, in (A)dS the same question can be rephrased as the question whether a cubic interaction $C(Y, Z)$ can be expressed making use of $\tilde{K}\left(Y_{\ell}, H_{12}, H_{23}, H_{31}\right)$ or not. More precisely, depending on the possible values of $\ell$, such coupling leads to

- trivial deformation of the $i$-th gauge transformation: $\delta_{E_{i}}^{(1)}=0$, if and only if the reexpression is possible for $\ell=i+1$ or $i-1$;

- trivial deformation of gauge algebra: $\llbracket E_{i}, E_{j} \rrbracket^{(0)}=0$, if and only if the re-expression is possible for any $\ell=1,2,3$.

In this section, we investigate these conditions and show, in particular, how they can be interpreted as conditions on the maximum number of derivatives in the (A)dS couplings.

\subsection{Contraction of curls}

Before analyzing the couplings given by the operators $H_{i j}$ 's, let us make a few remarks on the physical meaning of the $H_{i j}$ 's. From their definitions (1.15), it is easy to notice that

\footnotetext{
${ }^{13}$ See $[37,38]$ for some examples of amplitudes involving such interactions. It would be interesting to clarify these examples from the viewpoint of global-symmetry algebra.

${ }^{14}$ The issue of locality may arise only at the quartic order.

${ }^{15}$ Other non-standard higher-derivative kinetic terms for HS fields have been studied in [66] together with their gauge symmetries.
} 
they can be built using matrix operators $\left[\partial_{X_{i}} \partial_{U_{i}}\right]$ with components:

$$
\left[\partial_{X_{i}} \partial_{U_{i}}\right]_{M N}=\partial_{X_{i}^{[M}} \partial_{U_{i}^{N]}} .
$$

These are nothing but the curl operators with respect to the $i$-the field, and they are manifestly gauge invariant. One can now consider gauge-invariant scalar operators by taking traces of such operators (that is, contracting different curls) as

$$
H_{i_{1} \cdots i_{n}}=\operatorname{Tr}\left(\left[\partial_{X_{i_{1}}} \partial_{U_{i_{1}}}\right] \cdots\left[\partial_{X_{i_{n}}} \partial_{U_{i_{n}}}\right]\right) .
$$

However, as $\left[\partial_{X} \partial_{U}\right]^{\otimes n}$ corresponds to the curvature operator of rank $2 n$, one can check that the only independent ones among $H_{i_{1} \cdots i_{n}}$ 's are

$$
H_{12}, H_{23}, H_{31}, H_{123} \text {. }
$$

Moreover, the square of $H_{123}$ reduces to a product of $H_{i j}$ 's as

$$
H_{123}^{2} \Phi_{123} \stackrel{\mathrm{TT}}{\approx} \frac{1}{8} H_{12} H_{23} H_{31} \Phi_{123},
$$

and $H_{123}$ itself can be expressed in terms of $H_{i j}$ as

$$
H_{123} \Phi_{123} \stackrel{\mathrm{TT}}{\approx} \frac{1}{2} Y_{i} H_{i+1 i+2} \Phi_{123},
$$

when acted on the three-local fields $\Phi_{123}\left(X_{1}, U_{1} ; X_{2}, U_{2} ; X_{3}, U_{3}\right)$ satisfying the homogeneity and tangentiality conditions on each of the points.

\section{2 $H$-couplings}

In this subsection, we closely look the $H$-couplings $\tilde{K}\left(Y_{\ell}, H_{12}, H_{23}, H_{31}\right)$, and show how many derivatives they contain. For that, we need to find an independent set of couplings which span the space of all $H$-couplings. Such a set can be obtained by considering the monomials (see appendix $\mathrm{C}$ of [46] for more details):

$$
\begin{gathered}
Y_{\ell}^{\sigma} \tilde{H}_{1}^{h_{1}} \tilde{H}_{2}^{h_{2}} \tilde{H}_{3}^{h_{3}} \\
\stackrel{\mathrm{TT}}{\approx} Y_{\ell}^{\sigma} \prod_{i=1}^{3}\left[\sum_{n_{i}=0}^{h_{i}}\left(\begin{array}{c}
h_{i} \\
n_{i}
\end{array}\right)\left[h_{i}-\epsilon_{\ell, i} \sigma\right]_{n_{i}}\left(\lambda Z_{i}\right)^{n_{i}}\left(Y_{i+1} Y_{i-1}\right)^{h_{i}-n_{i}}\right],
\end{gathered}
$$

where $\epsilon_{\ell, i}=2 \delta_{\ell, i}-1$ and $\tilde{H}_{i}{ }^{\prime}$ s are

$$
\tilde{H}_{1}:=-H_{23}, \quad \tilde{H}_{2}:=-H_{31}, \quad \tilde{H}_{3}:=-H_{12} .
$$

In eq. (6.6), we have also provided their expressions as functions of $Y$ and $Z$, making clear the counting of derivatives (the $Y_{i}$ 's contain one derivative while the $Z_{i}$ 's do not). The powers $\sigma, h_{1}, h_{2}, h_{3}$ of (6.6) are related to the spins by

$$
s_{i}=h_{i+1}+h_{i-1}+\delta_{i, \ell} \sigma .
$$

For the following analysis, it is also convenient to distinguish two cases - whether the spins satisfy a triangular inequality or not:

$$
s_{\max } \leq s_{\text {mid }}+s_{\text {min }} \quad \text { or } \quad s_{\max }>s_{\text {mid }}+s_{\min } .
$$

However, it will turn out that the second case can be considered as a special class of the first one. 
Triangular case: $s_{\max } \leq s_{\operatorname{mid}}+s_{\min }$. When the triangular inequality holds, from eq. (6.8), we can determine the $h_{i}$ 's in terms of the spins and $\sigma=v+2 n$, where $v:=\bmod _{2}\left(s_{1}+s_{2}+s_{3}\right)$ and the non-negative integer $n$ is bounded as

$$
n \leq n_{*}:=\min \left\{\frac{s_{\ell}+s_{\ell+1}-s_{\ell-1}}{2}, \frac{s_{\ell}+s_{\ell-1}-s_{\ell+1}}{2}\right\} .
$$

Hence, we obtain a class of couplings labelled by $n:^{16}$

$$
\left(Y_{\ell} \tilde{H}_{\ell}\right)^{v} Y_{\ell}^{2 n} \tilde{H}_{1}^{\left\lfloor\frac{s_{2}+s_{3}-s_{1}}{2}\right\rfloor+\epsilon_{\ell, 1} n} \tilde{H}_{2}^{\left\lfloor\frac{s_{3}+s_{1}-s_{2}}{2}\right\rfloor+\epsilon_{\ell, 2} n} \tilde{H}_{3}^{\left\lfloor\frac{s_{1}+s_{2}-s_{3}}{2}\right\rfloor+\epsilon_{\ell, 3} n},
$$

where $\lfloor x\rfloor:=\max \{m \in \mathbb{Z} \mid m \leq x\}$. The highest-derivative parts of these couplings are all the same: $Y_{1}^{s_{1}} Y_{2}^{s_{2}} Y_{3}^{s_{3}}$, and its number of derivatives is $s_{1}+s_{2}+s_{3}$ - the maximum possible value. By taking linear combinations of these $n_{*}+1$ couplings, we can make $n_{*}$ different couplings where the highest-derivative terms cancel. The new highest-derivative part of such couplings are again all the same: $Y_{1}^{s_{1}-1} Y_{2}^{s_{2}-1} Y_{3}^{s_{3}-1} G(Y, Z)$, and their number of derivatives is lowered by two: $s_{1}+s_{2}+s_{3}-2$. One can continue this procedure until to obtain a coupling whose highest-derivative part is $Y_{1}^{s_{1}-n_{*}} Y_{2}^{s_{2}-n_{*}} Y_{3}^{s_{3}-n_{*}} G(Y, Z)^{n_{*}}$ (with the number of derivative $s_{1}+s_{2}+s_{3}-2 n_{*}$ ). Hence, starting from the basis (6.11) of $H$ couplings, we can obtain a new basis where couplings have all different highest-derivative parts. The couplings whose number of highest derivatives is $s_{1}+s_{2}+s_{3}-2 n$ reads

$$
\begin{aligned}
Q_{\ell, s_{1} s_{2} s_{3}}^{[n]} & =q_{\ell, v}^{[n]}\left(Y_{\ell} \tilde{H}_{\ell}\right)^{v} \tilde{H}_{1}^{\left\lfloor\frac{s_{2}+s_{3}-s_{1}}{2}\right\rfloor-\bar{\delta}_{\ell, 1} n} \tilde{H}_{2}^{\left\lfloor\frac{s_{3}+s_{1}-s_{2}}{2}\right\rfloor-\bar{\delta}_{\ell, 2} n} \tilde{H}_{3}^{\left\lfloor\frac{s_{1}+s_{2}-s_{3}}{2}\right\rfloor-\bar{\delta}_{\ell, 3} n}, \\
& \approx Y_{1}^{s_{1}-n} Y_{2}^{s_{2}-n} Y_{3}^{s_{3}-n} G(Y, Z)^{n}+\mathcal{O}(\lambda),
\end{aligned}
$$

where $\bar{\delta}_{\ell, i}=1-\delta_{\ell, i}$ and $q_{\ell, \sigma}^{[n]}$ is given by

$$
\begin{aligned}
q_{\ell, \sigma}^{[n+1]}= & \left(\tilde{H}_{\ell+1} \tilde{H}_{\ell-1}-Y_{\ell}^{2} \tilde{H}_{\ell}\right) \times \\
& \times \sum_{k=0}^{n}\left(\begin{array}{l}
n \\
k
\end{array}\right) \frac{(\sigma+2 n+1)_{k}[\sigma+1]_{n-k}}{\lambda^{n+1}(\sigma+1)_{2 n+1}}\left(\tilde{H}_{\ell+1} \tilde{H}_{\ell-1}\right)^{k}\left(-Y_{\ell}^{2} \tilde{H}_{\ell}\right)^{n-k} .
\end{aligned}
$$

To iterate, any $H$-coupling $\tilde{K}\left(Y_{\ell}, H_{12}, H_{23}, H_{31}\right)$ can be decomposed in terms of $Q_{\ell}^{[n]}$, ${ }^{17}$ with $n=0,1, \ldots, n_{*}$, and the value $n_{*}$ depends on $\ell$ (6.10). To be more concrete, let us assume without loss of generality

$$
s_{1} \geq s_{2} \geq s_{3} .
$$

Then, all possible $H$-couplings are given by

$$
Q_{1}^{[n]} \quad\left[n \leq \frac{s_{1}+s_{3}-s_{2}}{2}\right] ; \quad Q_{2}^{[n]}, Q_{3}^{[n]} \quad\left[n \leq \frac{s_{2}+s_{3}-s_{1}}{2}\right] .
$$

From the above, we can conclude the following:

- Non-deforming couplings

\footnotetext{
${ }^{16}$ The $n=0$ case is special since we can apply the identity (6.5) to replace $Y_{\ell} H_{\ell+1 \ell-1}$ with $H_{123}$. Involving only $H_{i j}$ 's and $H_{123}$, this coupling correspond to the full contraction of maximum curls, namely three linearized curvatures. So, it is the Born-Infeld coupling.

${ }^{17}$ Herefrom, we drop the subscript $s_{1} s_{2} s_{3}$ for compactness sake.
} 
First, in the range

$$
n \leq \frac{s_{2}+s_{3}-s_{1}}{2} \Rightarrow \#_{\partial} \geq 2 s_{1},
$$

all three $Q_{1}^{[n]}, Q_{2}^{[n]}$ and $Q_{3}^{[n]}$ are available. In fact, $Q_{2}^{[n]} \stackrel{\mathrm{TT}}{\approx} Q_{3}^{[n]}$ and they can be expressed as linear combinations of $Q_{1}^{[n]}$ 's. ${ }^{18}$ Hence, the couplings given by $Q_{2}^{[n]}$ 's or $Q_{3}^{[n]}$,s leave all three gauge transformations undeformed: $\delta_{E_{1,2,3}}^{(1)}=0$.

- Deforming Abelian couplings

In the range:

$$
\frac{s_{2}+s_{3}-s_{1}}{2}<n \leq \frac{s_{1}+s_{3}-s_{2}}{2} \Rightarrow 2 s_{2} \leq \# \#_{\partial}<2 s_{1},
$$

only $Q_{1}^{[n]}$ is available but neither $Q_{2}^{[n]}$ nor $Q_{3}^{[n]}$. Hence, the couplings given by $Q_{1}^{[n]}$ 's with $n$ in (6.18) necessarily deform the gauge transformation $\delta_{E_{1}}^{(1)} \neq 0$, but leave the other transformations and gauge algebra undeformed: $\delta_{E_{2,3}}^{(1)}=0$ and $\llbracket E_{i}, E_{j} \rrbracket^{(0)}=0$ for all $i$ and $j$.

- Non-Abelian couplings

Finally, in the range:

$$
n>\frac{s_{1}+s_{3}-s_{2}}{2} \Rightarrow \#_{\partial}<2 s_{2},
$$

all three $Q_{1}^{[n]}, Q_{2}^{[n]}$ and $Q_{3}^{[n]}$ are not available. Hence, any coupling, whose number of highest derivatives is smaller than $2 s_{2}$, cannot be written as a $H$-coupling, and deforms in (A)dS all three gauge transformations and all gauge algebra commutators: $\delta_{E_{1,2,3}}^{(1)} \neq 0$ and $\llbracket E_{i}, E_{j} \rrbracket^{(0)} \neq 0$.

This result is summarized in table 1 , and one can also count the numbers of couplings in the above three different classes:

$$
\begin{aligned}
(\# \text { of non-deforming couplings }) & =\left\lfloor\frac{1}{2}\left(s_{\min }+s_{\text {mid }}-s_{\max }\right)\right\rfloor+1, \\
(\# \text { of deforming Abelian couplings }) & =s_{\max }-s_{\text {mid }}, \\
(\# \text { of non-Abelian couplings }) & =\left\lceil\frac{1}{2}\left(s_{\min }+s_{\text {mid }}-s_{\max }\right)\right\rceil,
\end{aligned}
$$

where $\lfloor x\rfloor=\max \{m \in \mathbb{Z} \mid m \leq x\}$ and $\lceil x\rceil=\min \{n \in \mathbb{Z} \mid n \geq x\}$.

Non-triangular case: $s_{\max }>s_{\operatorname{mid}}+s_{\min }$. Let us again assume $s_{1} \geq s_{2} \geq s_{3}$. In this case, eq. (6.8) admit solutions only when $\ell=1$, and the corresponding $H$-couplings can be decomposed by

$$
Q_{1, s_{1} s_{2} s_{3}}^{[n]}=q_{1, s_{1}-s_{2}-s_{3}}^{[n]} Y_{1}^{s_{1}-s_{2}-s_{3}} \tilde{H}_{3}^{s_{2}-n} \tilde{H}_{2}^{s_{3}-n},
$$

\footnotetext{
${ }^{18}$ For the proof, consider the coupling:$$
C=Q_{2}^{[n]}-Q_{1}^{[n]}-c Q_{1}^{[n+1]}-\cdots-c^{\prime} Q_{1}^{\left.\left[\left(s_{1}+s_{3}-s_{2}\right) / 2\right]\right]},
$$

where the coefficients $c, \ldots, c^{\prime}$ are chosen such that the number of derivatives in $C$ is the lowest possible. Since $C$ gives $\delta_{E_{3}}^{(1)}=0$, it can be expressed either by $Q_{1}^{[m]}$ 's or by $Q_{2}^{[m]}$ 's, but the number of derivatives of $C$ is too low to allow them. Hence, one can conclude that $C$ must be zero, that is, any $Q_{2}^{[n]}$ can be written as $Q_{1}^{[m]}$ 's. One can do the same for $Q_{3}^{[n]}$ to conclude the same.
} 


\begin{tabular}{|c|c|c|c|c|c|}
\hline$n$ & $\#_{\partial}$ & $\delta_{E_{1}}^{(1)}$ & $\delta_{E_{2}}^{(1)}$ & $\delta_{E_{3}}^{(1)}$ & $C^{(3)}$ \\
\hline 0 & $s_{1}+s_{2}+s_{3}$ & $\neq 0$ & $=0$ & $=0$ & \\
$\vdots$ & $\vdots$ & $\vdots$ & $\vdots$ & $\vdots$ & $\approx \tilde{K}\left(Y_{1}, H_{12}, H_{23}, H_{31}\right)$ \\
$s_{3}$ & $s_{1}+s_{2}-s_{3}$ & $\vdots$ & $\vdots$ & $\vdots$ & \\
\hline
\end{tabular}

Table 4. Classification of cubic interactions $\left(s_{1} \geq s_{2} \geq s_{3}\right)$ in the non-triangular case $\left(s_{1}>s_{2}+s_{3}\right)$ according to the deformations of gauge transformations and gauge algebras.

\begin{tabular}{|c|c|c|c|c|}
\hline$n$ & $\#_{\partial}$ & $\delta_{E_{1}}^{(1)}$ & $\delta_{E_{2}}^{(1)}$ & $\delta_{E_{3}}^{(1)}$ \\
\hline 0 & 7 & 0 & 0 & 0 \\
1 & 5 & $*$ & 0 & 0 \\
2 & 3 & $*$ & $*$ & $*$ \\
\hline
\end{tabular}

\begin{tabular}{|c|c|c|c|c|}
\hline$n$ & $\#_{\partial}$ & $\delta_{E_{1}}^{(1)}$ & $\delta_{E_{2}}^{(1)}$ & $\delta_{E_{3}}^{(1)}$ \\
\hline 0 & 8 & 0 & 0 & 0 \\
1 & 6 & 0 & 0 & 0 \\
2 & 4 & $*$ & $*$ & $\Lambda$ \\
\hline
\end{tabular}

Table 5. 3-2-2 interactions

Table 6. $3-3-2$ interactions

where $n=0,1, \ldots, s_{3}$. Hence, in the non-triangular case, all the consistent couplings can be expressed in terms of $Q_{1}^{[n]}$,s so that they do not deform $\delta_{E_{2,3}}^{(1)}$ but deform $\delta_{E_{1}}^{(1)}$. This result is summarized in table 4 .

In this section, we have shown how the $H$-couplings are related to the curl of fields, and that in order to reproduce all couplings in Class I and II, we need to take particular combinations of such functions where the highest-derivative terms cancel. From the former point, one can notice that such couplings admit expressions in terms of HS curvatures. Let us notice that the construction of this kind of couplings making use of curvatures and of the cancellation of the highest-derivative piece were also discussed in [41].

\subsection{Examples}

Finally, for concreteness sake, let us consider two examples: $3-2-2$ and $3-3-2$ interactions. The table 1 reduces then to table 5 and 6 .

3-2-2 interactions. Any gauge invariant cubic vertices of 3-2-2 interactions can be expanded in the basis of $P_{322}^{[n]}$ 's $(2.7)$ as

$$
C(Y, Z)=k_{0} P^{[0]}+k_{1} P^{[1]}+k_{2} P^{[2]},
$$

where we have omitted the subscript 322 , and the $P^{[n]}$ 's are given by

$$
\begin{aligned}
P^{[0]}= & Y_{1}^{3} Y_{2}^{2} Y_{3}^{2}+2 \lambda Y_{1}^{2} Y_{2} Y_{3}\left(2 Y_{1} Z_{1}+3 Y_{2} Z_{2}+3 Y_{3} Z_{3}\right) \\
& +2 \lambda^{2} Y_{1}\left[\left(Y_{1} Z_{1}\right)^{2}+3\left(Y_{2} Z_{2}\right)^{2}+3\left(Y_{3} Z_{3}\right)^{2}+6 Y_{1} Z_{1}\left(Y_{2} Z_{2}+Y_{3} Z_{3}\right)+12 Y_{2} Z_{2} Y_{3} Z_{3}\right] \\
& +12 \lambda^{3}\left(2 Y_{1} Z_{1}+Y_{2} Z_{2}+Y_{3} Z_{3}\right) Z_{2} Z_{3}, \\
P^{[1]}= & Y_{1}^{2} Y_{2} Y_{3}\left(Y_{1} Z_{1}+Y_{2} Z_{2}+Y_{3} Z_{3}\right) \\
& +\lambda Y_{1}\left[\left(Y_{1} Z_{1}\right)^{2}+2\left(Y_{2} Z_{2}\right)^{2}+2\left(Y_{3} Z_{3}\right)^{2}+4 Y_{1} Z_{1}\left(Y_{2} Z_{2}+Y_{3} Z_{3}\right)+6 Y_{2} Z_{2} Y_{3} Z_{3}\right] \\
& +4 \lambda^{2}\left(2 Y_{1} Z_{1}+Y_{2} Z_{2}+Y_{3} Z_{3}\right) Z_{2} Z_{3} \\
P^{[2]}= & Y_{1}\left(Y_{1} Z_{1}+Y_{2} Z_{2}+Y_{3} Z_{3}\right)^{2}+2 \lambda\left(2 Y_{1} Z_{1}+Y_{2} Z_{2}+Y_{3} Z_{3}\right) Z_{2} Z_{3} .
\end{aligned}
$$


On the other hand, $H$-couplings provide a non-deforming and a deforming Abelian vertices, $Q_{\ell}^{[0]}$ and $Q_{1}^{[1]}$. Being gauge invariant, they can be also expressed as the expansion (6.22):

$$
\begin{aligned}
& Q_{\ell}^{[0]}=Y_{\ell} \tilde{H}_{\ell} \tilde{H}_{2} \tilde{H}_{3} \approx P^{[0]}-4 \lambda P^{[1]}+2 \lambda^{2} P^{[2]}, \\
& Q_{1}^{[1]}=q_{1,1}^{[1]} Y_{1} \tilde{H}_{1}=\lambda^{-1}\left(Y_{1}^{3} \tilde{H}_{1}^{2}-Y_{1} \tilde{H}_{1} \tilde{H}_{2} \tilde{H}_{3}\right) \approx P^{[1]}-2 \lambda P^{[2]} .
\end{aligned}
$$

These correspond to two vectors in the linear span of (6.23), and the remaining independent vector $P^{[2]}$ represents the non-Abelian coupling of $3-2-2$ interactions.

$\mathbf{3}-\mathbf{3}-\mathbf{2}$ interactions. In the case of $3-3-2$ interactions, we have

$$
\begin{aligned}
P^{[0]}= & Y_{1}^{3} Y_{2}^{3} Y_{3}^{2}+3 \lambda Y_{1}^{2} Y_{2}^{2} Y_{3}\left(2 Y_{1} Z_{1}+2 Y_{2} Z_{2}+3 Y_{3} Z_{3}\right) \\
& +6 \lambda^{2} Y_{1} Y_{2}\left[\left(Y_{1} Z_{1}\right)^{2}+\left(Y_{2} Z_{2}\right)^{2}+3\left(Y_{3} Z_{3}\right)^{2}+3 Y_{1} Z_{1} Y_{2} Z_{2}+6\left(Y_{1} Z_{1}+Y_{2} Z_{2}\right) Y_{3} Z_{3}\right] \\
& +6 \lambda^{3} Z_{3}\left[3\left(Y_{1} Z_{1}\right)^{2}+3\left(Y_{2} Z_{2}\right)^{2}+\left(Y_{3} Z_{3}\right)^{2}+12 Y_{1} Z_{1} Y_{2} Z_{2}+6\left(Y_{1} Z_{1}+Y_{2} Z_{2}\right) Y_{3} Z_{3}\right] \\
& +36 \lambda^{4} Z_{1} Z_{2} Z_{3}^{2}, \\
P^{[1]}= & Y_{1}^{2} Y_{2}^{2} Y_{3}\left(Y_{1} Z_{1}+Y_{2} Z_{2}+Y_{3} Z_{3}\right) \\
& +\lambda Y_{1} Y_{2}\left[2\left(Y_{1} Z_{1}\right)^{2}+2\left(Y_{2} Z_{2}\right)^{2}+4\left(Y_{3} Z_{3}\right)^{2}+5 Y_{1} Z_{1} Y_{2} Z_{2}+8\left(Y_{1} Z_{1}+Y_{2} Z_{2}\right) Y_{3} Z_{3}\right] \\
& +2 \lambda^{2} Z_{3}\left[3\left(Y_{1} Z_{1}\right)^{2}+3\left(Y_{2} Z_{2}\right)^{2}+\left(Y_{3} Z_{3}\right)^{2}+10 Y_{1} Z_{1} Y_{2} Z_{2}+5\left(Y_{1} Z_{1}+Y_{2} Z_{2}\right) Y_{3} Z_{3}\right] \\
& +10 \lambda^{3} Z_{1} Z_{2} Z_{3}^{2}, \\
P^{[2]}= & Y_{1} Y_{2}\left(Y_{1} Z_{1}+Y_{2} Z_{2}+Y_{3} Z_{3}\right)^{2} \\
& +\lambda Z_{3}\left[3\left(Y_{1} Z_{1}\right)^{2}+3\left(Y_{2} Z_{2}\right)^{2}+\left(Y_{3} Z_{3}\right)^{2}+8 Y_{1} Z_{1} Y_{2} Z_{2}+4\left(Y_{1} Z_{1}+Y_{2} Z_{2}\right) Y_{3} Z_{3}\right] \\
& +4 \lambda^{2} Z_{1} Z_{2} Z_{3}^{2} .
\end{aligned}
$$

On the other hand, $H$-couplings provide two non-deforming vertices, $Q_{\ell}^{[0]}$ and $Q_{\ell}^{[1]}$, which can be expressed as

$$
\begin{aligned}
& Q_{\ell}^{[0]}=\tilde{H}_{1} \tilde{H}_{2} \tilde{H}_{3}^{2} \approx P^{[0]}-5 \lambda P^{[1]}+4 \lambda^{2} P^{[2]}, \\
& Q_{\ell}^{[1]}=q_{\ell, 0}^{[1]} \tilde{H}_{\ell} \tilde{H}_{3}=\lambda^{-1}\left(Y_{\ell}^{2} \tilde{H}_{\ell}^{2} \tilde{H}_{3}-\tilde{H}_{1} \tilde{H}_{2} \tilde{H}_{3}^{2}\right) \approx P^{[1]}-2 \lambda P^{[2]} .
\end{aligned}
$$

Again, the other independent coupling $P^{[2]}$ represents the non-Abelian 3-3-2 interaction.

\section{Discussions}

In the present paper, we have analysed the deformations of both gauge transformations and gauge algebras induced by the cubic couplings of massless fields constructed in [42]. The main results of the paper are already summarized by table 1, but let us rephrase them once again here:

- Defining $\Sigma_{i}=s_{i}$ in flat space and $\Sigma_{i}=\max \left\{s_{i}, s_{\text {mid }}\right\}$ in $(\mathrm{A}) \mathrm{dS}$, the highest number derivatives $\#_{\partial}$ of a coupling with $\delta_{E_{i}}^{(1)}=0$ satisfies

$$
\text { (\#ə of a coupling with } \left.\delta_{E_{i}}^{(1)}=0\right) \geq 2 \Sigma_{i} .
$$

Equivalently, if the highest-derivative term of a cubic interaction involves less than $2 \Sigma_{i}$ derivatives, then the coupling necessarily induces a non-trivial deformation of gauge transformation: $\delta_{E_{i}}^{(1)} \neq 0$. 
- Defining $\Sigma_{i j}=\min \left\{s_{i}, s_{j}\right\}$ in flat space and $\Sigma_{i j}=s_{\text {mid }}$ in (A)dS, the highest number of derivatives $\#_{\partial}$ in a coupling with $\llbracket E_{i}, E_{j} \rrbracket^{(0)}=0$ satisfies

$$
\text { (\# } \left.\text { \# }_{\partial} \text { a coupling with } \llbracket E_{i}, E_{j} \rrbracket^{(0)}=0\right) \geq 2 \Sigma_{i j} \text {. }
$$

Equivalently, if the highest-derivative terms of a cubic interaction involves less than $2 \Sigma_{i j}$ derivatives, then the coupling necessarily induces a non-Abelian deformation of the gauge algebra: $\llbracket E_{i}, E_{j} \rrbracket^{(0)} \neq 0$.

This is the first classification of cubic interactions according to the deformations of each bracket $\llbracket E_{i}, E_{j} \rrbracket^{(0)}$ and each gauge transformation $\delta_{E_{i}}^{(1)}$. As a result, we categorized all cubic interactions into four different classes (see table 1). ${ }^{19}$ Class I and IV correspond to the set of couplings which deform nothing and everything, respectively. The other two classes have some interesting features. Class II couplings leave the gauge algebra invariant but deform one out of three gauge transformations. This means that the other two fields are charged with respect to the last field, and should correspond the couplings responsible for HS-algebra multiplets, together with the massless-massive-massive cubic interactions (see below). Class III couplings induce non-trivial deformations of gauge transformations for all three fields (hence for all three brackets) in (A)dS, but one of them (two of them, considering brackets) vanishes in the flat-space limit. The gravitational interactions of HS fields belong to this class, and the corresponding gauge transformations, which vanish in the flat-space limit, are the general coordinate transformation (or diffeomorphism) of HS fields. The couplings belonging to Class III have also interesting implications on the global symmetries: a conclusion similar to the Coleman-Mandula theorem can be derived in our setting as a consequence of the Jacobi identity.

$H$ - and $G$-couplings. In the case of (A)dS interactions, we have shown that the classifications of couplings can be entirely rephrased in terms of the possibility of rewriting them as $H$-couplings. Hence, the classification boils down to the question whether a $G$-coupling (the coupling given by $Y_{i}$ 's and $G$ ) is expressible as a $H$-coupling (the coupling given by $H_{i j}$ 's and one of $Y_{i}$ 's) or not. Although, our aim was to analyze the three-massless-fields interactions, let us briefly discuss the interactions of one massless (gauge) field and two massive (matter) fields, where the distinction between $H$ - and $G$-coupling become more clear. The general solution of the equation (2.3) consists in two parts:

$$
C(Y, Z)=C_{H}(Y, Z)+\delta_{\mu_{2}-\mu_{3} \in 2 \mathbb{Z}} C_{G}(Y, Z) .
$$

\footnotetext{
${ }^{19}$ At this point, let us summarize once again what was known before about the general classification of the HS gauge-symmetry deformations. The results can be categorized into two groups - the metric-like formulation for flat space interactions, and the frame-like formulation for strictly (A)dS interactions. In the former case, the minimum number of derivatives of cubic interactions with $\llbracket E_{1}, E_{2} \rrbracket^{(0)}=\llbracket E_{2}, E_{3} \rrbracket^{(0)}=$ $\llbracket E_{3}, E_{1} \rrbracket^{(0)}=0$ has been identified in [25], but neither for each of the $\llbracket E_{i}, E_{j} \rrbracket^{(0)}$ 's nor for the $\delta_{E_{i}}^{(1)}$ 's. In the latter case, the non-Abelian vertices, corresponding in our classification to the Class III and IV couplings, have been constructed in [22] together with the discussions on the Abelian and the Current vertices, which correspond respectively to the Class I and II couplings.
} 

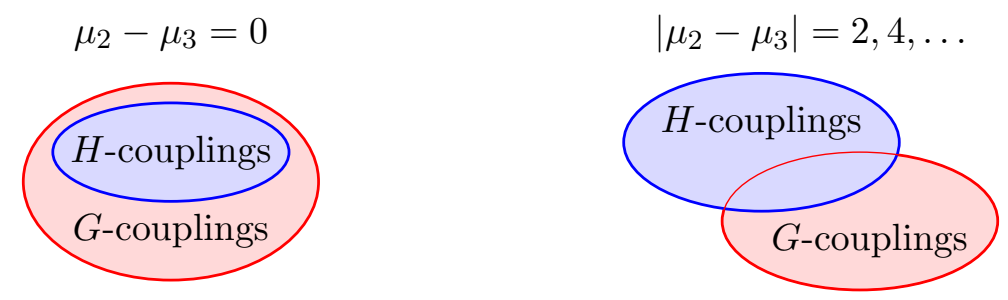

Figure 1. Schematic diagram of the relation between $H$ - and $G$-couplings

The solutions $C_{H}$ correspond to what we call $H$-couplings. Their key property is the existence of a field redefinition bringing their form to

$$
C_{H}(Y, Z) \approx \tilde{K}\left(Z_{1}, Y_{2}, Y_{3}, H_{12}, H_{13}\right),
$$

that it is manifestly off-shell gauge invariant. On the other hand, the $G$-couplings $C_{G}$ are present only when $\mu_{2}-\mu_{3} \in 2 \mathbb{Z}$, and they may lead to deformations of gauge symmetries. Their form is given by

$$
C_{G}(Y, Z)=\left.Y_{2}^{R\left(\frac{\mu_{2}-\mu_{3}}{2}\right)} Y_{3}^{R\left(\frac{\mu_{3}-\mu_{2}}{2}\right)} e^{\lambda \mathcal{D}} K\left(Z_{1}, Y_{1}, Y_{2}, Y_{3}, G\right)\right|_{G=G(Y, Z)},
$$

where $R(x)=(x+|x|) / 2$ is the ramp function. The latter $H$ - and $G$-couplings are not totally independent, and have a non-vanishing overlap. In particular, when $\mu_{2}-\mu_{3}=0$, all the $H$-couplings can be written as $G$-couplings (see figure 1 ). The physical interpretation of these class of couplings becomes more transparent when one rewrites them as a coupling between a gauge field and a conserved current bilinear in matter fields. The question whether the deformation is trivial or not becomes the question whether the current is identically conserved or not. The $H$-couplings (7.4) provide us with the full list of interactions expressible using identically conserved currents, namely improvements. On the contrary, the couplings in the coset $G / H$ correspond to those associated to on-shell conserved currents, namely Noether currents. It is interesting to notice that Noether currents in (A)dS can involve fields with different masses contrary to the flat-space case. This means that HS multiplets consisting of matter fields may involve fields of different masses.

As we mentioned, to identify the couplings associated with Noether currents, one should quotient the space of $G$-solutions (7.5) by that of $H$-solutions (7.4). This can be conveniently done by rewriting them in a common basis (see [46] for more details):

$$
\begin{aligned}
P_{\sigma_{1} \sigma_{2} \sigma_{3} v}^{\tau_{1}}\left(\bar{\mu}_{1} ; Y, Z\right)=\sum_{p, q \geq 0}^{p+q \leq \sigma_{1}} & \frac{\left[\sigma_{2}+\frac{\bar{\mu}_{1}}{2}\right]_{p}\left[\sigma_{3}-\frac{\bar{\mu}_{1}}{2}\right]_{q}}{\left[\sigma_{2}+\sigma_{3}\right]_{p+q}} \frac{\left(\lambda Z_{3} \partial_{Y_{1}} \partial_{Y_{2}}\right)^{p}}{p !} \frac{\left(\lambda Z_{2} \partial_{Y_{3}} \partial_{Y_{1}}\right)^{q}}{q !} \times \\
& \times Z_{1}^{\tau_{1}} Y_{1}^{\sigma_{1}} Y_{2}^{\sigma_{2}} Y_{3}^{\sigma_{3}}\left[G_{1}(Y, Z)\right]^{v}
\end{aligned}
$$

where $[a]_{n}:=a(a-1) \cdots(a-n+1)$ is the descending Pochhammer symbol and $\bar{\mu}_{1}=\mu_{3}-$ $\mu_{2} \in 2 \mathbb{Z}$. The spins are related to the indices $\sigma_{i}, \tau_{1}$ and $v$ by $s_{1}=\sigma_{1}+v, s_{2}=\sigma_{2}+\tau_{1}+v$ and $s_{3}=\sigma_{3}+\tau_{1}+v$. In this basis, the genuine Noether couplings correspond to the ones with $\sigma_{2}+\sigma_{3}<\sigma_{1}$. Let us also stress that the allowed mass difference is bounded by spins as $-2 s_{3} \leq \bar{\mu}_{1} \leq 2 s_{2}$. The corresponding gauge-transformation deformation $\delta_{E_{1}}^{(1)}$ of the massive fields are given by the general formula (3.7). 
Partially-massless fields. The simple pattern of the non-deforming cubic interactions of massless fields suggests its straightforward generalization to the partially-massless (PM) interactions: the couplings which do not deform any gauge transformation (the analog of Class I) have the form:

$$
C=\sum_{\sigma_{l-1}=0}^{\mu_{l-1}} \sum_{\sigma_{l+1}=0}^{\mu_{l+1}} Y_{l-1}^{\sigma_{l-1}} Y_{l+1}^{\sigma_{l+1}} \tilde{K}^{\sigma_{l-1} \sigma_{l+1}}\left(Y_{l}, \tilde{H}_{1}, \tilde{H}_{2}, \tilde{H}_{3}\right), \quad(l=2,3),
$$

while the couplings which deform only one of three gauge transformation but no bracket (the analog of Class II) have the form:

$$
C=\sum_{\sigma_{2}=0}^{\mu_{2}} \sum_{\sigma_{3}=0}^{\mu_{3}} Y_{2}^{\sigma_{2}} Y_{3}^{\sigma_{3}} \tilde{K}^{\sigma_{2} \sigma_{3}}\left(Y_{1}, \tilde{H}_{1}, \tilde{H}_{2}, \tilde{H}_{3}\right)
$$

This conjecture, if true, completes the classification of the PM cubic interactions. We do not need to consider a possible distinction like the massless Class III and IV, since PM representations themselves decomposes into standard massless ones in the flat-space limit. In this way, non-Abelian interactions can be identified simply by the couplings which cannot be written as above.

\section{Acknowledgments}

We thank N. Boulanger, M. Henneaux, K. Mkrtchyan and E. Skvortsov for useful discussions. We performed various computations with xAct [69] and xTras [70] pagkages for Mathematica, and we are grateful to T. Nutma for his advices. We also acknowledge the GGI, Florence workshop on "Higher spins, Strings and Duality" where the present work was developed.

\section{A Transverse and traceless part}

In the settings of our works [42, 43, 46], one of the most important ingredients is to consider the TT part of the vertices separately from the other parts. This enabled us to simplify the technically involved problem of constructing consistent HS interactions into small steps, of which the first one - constructing the TT part of the vertices - could be solved independently from the others. In this appendix, we explain this point by recalling the demonstration provided in [44].

In the analysis of the gauge invariance condition $\delta S=0$, it is convenient to split the interacting action $S$ into two parts: the one which does not involve any divergence, trace or auxiliary fields (TT part), and the one which does (DTA part). The point is that the TT part of the vertex can be determined without using any information about the other part. First, let us note that any functional $S$ can be separated in a unique way into its TT part and the DTA part as

$$
S=[S]_{\mathrm{TT}}+[S]_{\mathrm{DTA}}
$$


after removing all the ambiguities given by integrations by parts or field redefinitions. Moreover, the gauge invariance condition can be also split into two equations:

$$
\left[\delta^{(0)} S^{(3)}\right]_{\mathrm{TT}} \approx 0, \quad\left[\delta^{(0)} S^{(3)}\right]_{\mathrm{DTA}} \approx 0 .
$$

Second, as the gauge variations of divergences, traces or auxiliary fields are proportional to themselves up to terms proportional to $\square$ as

$$
\left[\delta^{(0)}\left[S^{(3)}\right]_{\mathrm{DTA}}\right]_{\mathrm{TT}} \approx 0,
$$

the first equation in (A.2) provides an independent condition for the TT parts, $\left[S^{(3)}\right]_{\mathrm{TT}}$, of the interactions:

$$
\left[\delta^{(0)} S^{(3)}\right]_{\mathrm{TT}}=\left[\delta^{(0)}\left\{\left[S^{(3)}\right]_{\mathrm{TT}}+\left[S^{(3)}\right]_{\mathrm{DTA}}\right\}\right]_{\mathrm{TT}} \approx\left[\delta^{(0)}\left[S^{(3)}\right]_{\mathrm{TT}}\right]_{\mathrm{TT}} \approx 0
$$

This analysis is valid for any homogeneous condition $\delta^{(0)} S^{(n)} \approx 0$ of any order $n$ since $\left[\delta^{(0)}\left[S^{(n)}\right]_{\mathrm{DTA}}\right]_{\mathrm{TT}} \approx 0$. However, it is important to notice that eventual divergence terms might contribute to the inhomogeneous conditions (1.2) when combined with higher-order deformations $\delta^{(n \geq 1)}$. Therefore, for higher-order interactions, more careful analysis is needed. We hope to report on this issue in the near future.

In below, we provide a few more remarks on the issues of restricting to the TT part:

- Let us recall here an observation made in section 5: as far as the global-symmetry algebra is concerned, the restriction to the TT part does not filter any information since gauge parameters associated to global symmetries already satisfy the TT conditions.

- Let us emphasize once again that in this TT set up, we are not imposing any gauge condition. Therefore, the deformation $\delta^{(1)} \Phi$ is not required to satisfy any condition such as tracelessness or transversality. Instead, the consequence of the TT-part restriction is that we get only a part — but all physically relevant — of the information on $\delta^{(1)} \Phi$, as discussed in the paragraphs after eq. (3.9).

- Finally, let us mention that there is no vertex whose TT part does not induce any deformation while its other part does. It is because our analysis is based on whether a given vertex can be re-expressed into a $H$ coupling, whose gauge invariance properties do not rely on any TT conditions.

\section{B Constants generated by $\lambda$}

In this appendix, we explain how to eliminate $\lambda$ in terms of proper factors of $\Lambda$. The $\lambda$, which was denoted by $-\hat{\delta} / L$ in our previous papers, is defined to play the following role inside of the $(\mathrm{A}) \mathrm{dS}$ action:

$$
\int_{(\mathrm{A}) \mathrm{dS}} \lambda^{n} I_{\Delta}=\int d^{d+1} X \delta^{[n]}\left(\sqrt{\epsilon X^{2}}-L\right)\left(\frac{-\epsilon}{L}\right)^{n} I_{\Delta}
$$


where $I_{\Delta}$ is an integrand of the homogeneity degree $\Delta$. The sign $\epsilon$ is positive for dS and negative for $\operatorname{AdS}$ and $\delta^{[n]}$ is the $n$-th derivative of the delta distribution. Hence, after integrating by part the radial variable, one obtains

$$
\int_{(\mathrm{A}) \mathrm{dS}} \lambda^{n} I_{\Delta}=(\Delta+d-1)(\Delta+d-3) \cdots(\Delta+d-2 n+1) \Lambda^{n} \int_{(\mathrm{A}) \mathrm{dS}} I_{\Delta},
$$

with the cosmological constant $\Lambda=\epsilon / L^{2}$. Therefore, one can see that $\lambda^{n}$ inside of action generates $\Lambda^{n}$ with the above constant which depends on the space-time dimensionality $d$ and the degree of homogeneity of the integrand $I_{\Delta}$. Although the above replacement is possible only inside of the action, we can still formally assume the above formulas, since the degree of homogeneity $\Delta$ is preserved through our analysis.

\section{Analysis of trivial deformations}

Let us first consider the following simple problem: for a given function $C$ whether there exists a solution $\Omega(t)$ for

$$
\left(t-Y_{1} \partial_{Z_{2}}+Y_{2} \partial_{Z_{1}}\right) \Omega\left(t ; Y_{1}, Y_{2}, Z_{1}, Z_{2}\right)=C\left(Y_{1}, Y_{2}, Z_{1}, Z_{2}\right) .
$$

To analyze this, we again expand $\Omega(t)$ in $t$ as

$$
\Omega\left(t ; Y_{1}, Y_{2}, Z_{1}, Z_{2}\right)=\sum_{k=0}^{\infty} t^{k} \omega_{k}\left(Y_{1}, Y_{2}, Z_{1}, Z_{2}\right),
$$

then the equation (C.1) reduces to

$$
\omega_{-1}=C, \quad \omega_{k-1}=\left(Y_{1} \partial_{Z_{2}}-Y_{2} \partial_{Z_{1}}\right) \omega_{k} \quad[k=0,1,2, \ldots] .
$$

Since the differential operator commutes with the number operators:

$$
N_{1}=Y_{1} \partial_{Y_{1}}+Z_{2} \partial_{Z_{2}}, \quad N_{2}=Y_{2} \partial_{Y_{2}}+Z_{1} \partial_{Z_{1}},
$$

while have eigenvalues -1 with

$$
N_{Z}=Z_{1} \partial_{Z_{1}}+Z_{2} \partial_{Z_{2}}
$$

we can address this problem in the subspace:

$$
C \in V^{\left(n_{1}, n_{2}, n_{z}-1\right)}
$$

where

$$
\left(N_{1}, N_{2}, N_{Z}\right) V^{\left(n_{1}, n_{2}, n_{z}\right)}=\left(n_{1}, n_{2}, n_{z}\right) V^{\left(n_{1}, n_{2}, n_{z}\right)},
$$

then the solution belongs to

$$
\omega_{k} \in V^{\left(n_{1}, n_{2}, n_{z}+k\right)} .
$$

The space $V^{\left(n_{1}, n_{2}, n_{z}\right)}$ is spanned by the monomials:

$$
p_{m}^{\left(n_{1}, n_{2}, n_{z}\right)}=Y_{1}^{n_{1}-n_{z}+m} Y_{2}^{n_{2}-m} \frac{Z_{1}^{m}}{m !} \frac{Z_{2}^{n_{z}-m}}{\left(n_{z}-m\right) !} .
$$


The image of $\Omega^{\left(n_{1}, n_{2}, n_{z}\right)}$ by the differential map is spanned by

$$
\left\{p_{m}^{\left(n_{1}, n_{2}, n_{z}-1\right)}-p_{m-1}^{\left(n_{1}, n_{2}, n_{z}-1\right)} \mid \max \left\{n_{z}-n_{1}, 0\right\} \leq m \leq \min \left\{n_{z}, n_{2}\right\}\right\}
$$

while the codomain, the space of $C^{\left(n_{1}, n_{2}, n_{z}-1\right)}$, is spanned by

$$
\left\{p_{m}^{\left(n_{1}, n_{2}, n_{z}-1\right)} \mid \max \left\{n_{z}-n_{1}-1,0\right\} \leq m \leq \min \left\{n_{z}-1, n_{2}\right\}\right\} .
$$

The two sets span the same space if and only if

$$
n_{z} \leq n_{1} \quad \text { or } \quad n_{z} \leq n_{2}
$$

In general we deal with funcitons

$$
C=\bigoplus C^{\left(n_{1}-n_{z}+k, n_{2}-n_{z}+k, k-1\right)}
$$

satisfy

$$
\tau_{1}+1 \leq \sigma_{1} \quad \text { or } \quad \tau_{2}+1 \leq \sigma_{2},
$$

then there always exists a solution $\Omega$.

\section{Counting of (A)dS structure constants}

In this appendix, we give more details on the counting of the number of independent (A)dS structure constants. As explained in the text, it is not difficult to see, using $\mathfrak{s p}_{2}$ commutation relations, that the general solution for the bracket is given by

$$
\mathcal{F}^{s_{3}}{ }_{s_{1} s_{2}}=e^{Z_{1}+Z_{2}} \mathfrak{F}^{s_{3}}{ }_{s_{1} s_{2}}\left(G_{3}, H_{3}\right) .
$$

In particular the generic structure constant $\mathcal{F}^{s_{3}} s_{1} s_{2}$ will be a linear combination of the following independent structures:

$$
Z_{1}^{r_{2}-v-h} Z_{2}^{r_{1}-v-h} G_{3}^{(v) r_{1} r_{2}} H_{3}^{h}, \quad r_{1}+r_{2}-v-2 h=r_{3},
$$

where we have used the short-hand notation:

$$
G_{3}^{(v) r_{1} r_{2}}=\sum_{k=0}^{v}\left(\begin{array}{l}
v \\
k
\end{array}\right) \frac{\left(Y_{1} Z_{1}\right)^{k}\left(Y_{2} Z_{2}\right)^{v-k}}{\left(r_{2}-v-h+k\right) !\left(r_{1}-h-k\right) !} .
$$

Hence, in order to count the independent terms (D.2) we need to find the number of solutions of

$$
v+2 h=s_{1}+s_{2}-s_{3}-1, \quad v+h \leq \min \left[s_{1}, s_{2}\right]-1,
$$

that is precisely the condition solved in the text. 


\section{E No-go for the spin-3 gravitational coupling in flat space}

In this appendix, we come back to the discussion on the flat-space gravitational coupling of HS fields of section 5. The only case left over is the possibility of allowing the bracket:

$$
\llbracket T_{3}, T_{2} \rrbracket \sim T_{2},
$$

induced by the three-derivative $2-2-3$ coupling. ${ }^{20}$ If such a bracket exists, then the r.h.s. of (5.40) with $s=s^{\prime}=3$ is not zero but $\sim T_{2}$ so that one cannot simply conclude $\llbracket T_{2}, T_{2} \rrbracket=0$. Before analyzing this case in more detail, let us notice that this case still excludes any gravitational interactions for spin $s \geq 4$ fields, and requires that gravitons be colored for the non-triviality of $2-2-3$ coupling - a very exotic scenario, which we shall rule out by the following analysis. Consider the spin-2 part of the Jacobi identity:

$$
\llbracket \llbracket \bar{E}_{1}^{(3)}, \bar{E}_{2}^{(3)} \rrbracket, \bar{E}_{3}^{(2)} \rrbracket+\llbracket \llbracket \bar{E}_{2}^{(3)}, \bar{E}_{3}^{(2)} \rrbracket, \bar{E}_{1}^{(3)} \rrbracket+\llbracket \llbracket \bar{E}_{3}^{(2)}, \bar{E}_{1}^{(3)} \rrbracket, \bar{E}_{2}^{(3)} \rrbracket=0,
$$

where the superscript $(n)$ indicates the spin of the associated field. While the last two terms get contributions only from the bracket induced by the three-derivative $2-2-3$ coupling (hence in total six derivatives), there exist three possible contributions for the first term:

1. the first bracket by the three-derivative $3-3-3$ coupling; and the second bracket by the three-derivative $2-2-3$ coupling (hence in total six derivatives);

2. the first bracket by the five-derivative $3-3-3$ couplings; and the second bracket by the three-derivative $2-2-3$ coupling (hence in total eight derivatives);

3. the first bracket by the four-derivative $2-3-3$ coupling; and the second bracket by the two-derivative $2-2-2$ coupling (hence in total six derivatives).

In fact, the first contribution cannot be considered since the three-derivative $3-3-3$ coupling, discovered longtime ago [12], has been shown to be inconsistent in [27]. The second contribution is neither possible since it involves eight derivatives differently from all other contributions. The only remaining possibility is the third case, and to examine it we need the explicit forms of the brackets:

$$
\llbracket \bar{E}_{1}, \bar{E}_{2} \rrbracket=\mathcal{X}_{\alpha}\left(c^{\alpha}{ }_{a b} G_{3} H_{3} \bar{E}_{1}^{a} \bar{E}_{2}^{b}+f_{\beta a}^{\alpha} H_{3} \bar{E}_{1}^{\beta} \bar{E}_{2}^{a}+d^{\alpha}{ }_{\beta \gamma} G_{3} \bar{E}_{1}^{\beta} \bar{E}_{2}^{\gamma}\right),
$$

where the Killing tensors $\bar{E}_{i}$ 's contain both the spin-2 and spin-3 parts with Chan-Paton factors labelled respectively by $\alpha, \beta$ and $a, b$ :

$$
\bar{E}_{i}=\mathcal{X}_{\alpha} \bar{E}_{i}^{\alpha}+\mathcal{Y}_{a} \bar{E}_{i}^{a} .
$$

The coefficients $c^{\alpha}{ }_{a b}, f^{\alpha}{ }_{\beta a}$ and $d^{\alpha}{ }_{\beta \gamma}$ are the corresponding structure constants of the internal symmetry. Plugging eq. (E.3) into the Jacobi identity (E.2), one gets

$$
\begin{aligned}
& \frac{1}{4}\left(f_{\gamma a}^{\alpha} f_{\beta b}^{\gamma}+f^{\alpha}{ }_{\gamma b} f_{\beta a}^{\gamma}\right) Z_{14}\left(Y_{12} Y_{21}-W_{12} Z_{12}+Y_{13} Y_{21}-W_{13} Z_{12}\right)\left(Y_{23} Y_{32}-W_{23} Z_{23}\right) \\
& +d^{\alpha}{ }_{\beta \gamma} c_{a b}^{\gamma}\left(Y_{12} Y_{21}-W_{12} Z_{12}\right)\left(Y_{12} Y_{32} Z_{24}+Y_{12} Y_{31} Z_{24}+Y_{13} Y_{21} Z_{34}\right. \\
& \left.-Y_{12} Y_{23} Z_{34}-Y_{21} Y_{31} Z_{14}-Y_{21} Y_{32} Z_{14}\right)=0,
\end{aligned}
$$

\footnotetext{
${ }^{20}$ Notice that all $2-2-s$ couplings are Abelian for $s>3$ so that the only case in which they might play any role for HS algebras is the $s=3$ case.
} 
where $\left(W_{i j}, Y_{i j}, Z_{i j}\right)=\left(\partial_{X_{i}} \cdot \partial_{X_{j}}, \partial_{U_{i}} \cdot \partial_{X_{j}}, \partial_{U_{i}} \cdot \partial_{U_{j}}\right)$, and the equality holds when it acts on the Killing tensors $\bar{E}_{1}^{a} \bar{E}_{2}^{b} \bar{E}_{3}^{\beta}$. Apparently, this expression as a function of the variables $W_{i j}, Y_{i j}, Z_{i j}$ 's is not identically zero. However, one should examine all possible identities of such variables due to the properties of Killing tensors. For that, we have evaluated the expression on the generators of the type:

$$
\bar{E}_{i}^{(n)}=\left[\left(W_{i}\right)_{M N} U^{M} X^{N}\right]^{n}
$$

with antisymmetric constant matrices $W_{i}$ 's. This has been carried out with the help of Mathematica and we found that the relation (E.5) cannot hold for non-trivial internal structure constants $c^{\alpha}{ }_{a b}, f^{\alpha}{ }_{\beta a}$ and $d^{\alpha}{ }_{\beta \gamma}$.

Open Access. This article is distributed under the terms of the Creative Commons Attribution License (CC-BY 4.0), which permits any use, distribution and reproduction in any medium, provided the original author(s) and source are credited.

\section{References}

[1] X. Bekaert, N. Boulanger and P. Sundell, How higher-spin gravity surpasses the spin two barrier: no-go theorems versus yes-go examples, Rev. Mod. Phys. 84 (2012) 987 [arXiv: 1007.0435] [INSPIRE].

[2] M.A. Vasiliev, Consistent equation for interacting gauge fields of all spins in (3+1)-dimensions, Phys. Lett. B 243 (1990) 378 [INSPIRE].

[3] M.A. Vasiliev, Properties of equations of motion of interacting gauge fields of all spins in (3+1)-dimensions, Class. Quant. Grav. 8 (1991) 1387 [INSPIRE].

[4] M.A. Vasiliev, Higher spin gauge theories in four-dimensions, three-dimensions and two-dimensions, Int. J. Mod. Phys. D 5 (1996) 763 [hep-th/9611024] [INSPIRE].

[5] M.A. Vasiliev, Nonlinear equations for symmetric massless higher spin fields in (A)dS(d), Phys. Lett. B 567 (2003) 139 [hep-th/0304049] [INSPIRE].

[6] X. Bekaert, S. Cnockaert, C. Iazeolla and M.A. Vasiliev, Nonlinear higher spin theories in various dimensions, hep-th/0503128 [INSPIRE].

[7] A.K.H. Bengtsson, I. Bengtsson and L. Brink, Cubic Interaction Terms for Arbitrary Spin, Nucl. Phys. B 227 (1983) 31 [inSPIRE].

[8] R.R. Metsaev, Generating function for cubic interaction vertices of higher spin fields in any dimension, Mod. Phys. Lett. A 8 (1993) 2413 [InSPIRE].

[9] E.S. Fradkin and R.R. Metsaev, Cubic scattering amplitudes for all massless representations of the Poincaré group in any space-time dimension, Phys. Rev. D 52 (1995) 4660 [INSPIRE].

[10] R.R. Metsaev, Cubic interaction vertices of massive and massless higher spin fields, Nucl. Phys. B 759 (2006) 147 [hep-th/0512342] [INSPIRE].

[11] R.R. Metsaev, Cubic interaction vertices for fermionic and bosonic arbitrary spin fields, Nucl. Phys. B 859 (2012) 13 [arXiv:0712.3526] [INSPIRE].

[12] F.A. Berends, G.J.H. Burgers and H. Van Dam, On spin three selfinteractions, Z. Phys. C 24 (1984) 247 [INSPIRE]. 
[13] Y. Zinoviev, On spin 3 interacting with gravity, Class. Quant. Grav. 26 (2009) 035022 [arXiv:0805.2226] [INSPIRE].

[14] Y. Zinoviev, Spin 3 cubic vertices in a frame-like formalism, JHEP 08 (2010) 084 [arXiv: 1007.0158] [INSPIRE].

[15] R. Manvelyan, K. Mkrtchyan and W. Ruehl, Direct Construction of A Cubic Selfinteraction for Higher Spin gauge Fields, Nucl. Phys. B 844 (2011) 348 [arXiv:1002.1358] [INSPIRE].

[16] R. Manvelyan, K. Mkrtchyan and W. Rühl, General trilinear interaction for arbitrary even higher spin gauge fields, Nucl. Phys. B 836 (2010) 204 [arXiv: 1003.2877] [INSPIRE].

[17] R. Manvelyan, K. Mkrtchyan and W. Ruehl, A Generating function for the cubic interactions of higher spin fields, Phys. Lett. B 696 (2011) 410 [arXiv:1009.1054] [INSPIRE].

[18] M. Taronna, Higher Spins and String Interactions, arXiv:1005.3061 [INSPIRE].

[19] A. Sagnotti and M. Taronna, String Lessons for Higher-Spin Interactions, Nucl. Phys. B 842 (2011) 299 [arXiv: 1006.5242] [INSPIRE].

[20] E.S. Fradkin and M.A. Vasiliev, Cubic Interaction in Extended Theories of Massless Higher Spin Fields, Nucl. Phys. B 291 (1987) 141 [INSPIRE].

[21] E.S. Fradkin and M.A. Vasiliev, On the Gravitational Interaction of Massless Higher Spin Fields, Phys. Lett. B 189 (1987) 89 [INSPIRE].

[22] M.A. Vasiliev, Cubic Vertices for Symmetric Higher-Spin Gauge Fields in $(A) d S_{d}$, Nucl. Phys. B 862 (2012) 341 [arXiv:1108.5921] [INSPIRE].

[23] N. Boulanger, D. Ponomarev and E.D. Skvortsov, Non-abelian cubic vertices for higher-spin fields in anti-de Sitter space, JHEP 05 (2013) 008 [arXiv: 1211.6979] [INSPIRE].

[24] N. Boulanger, S. Leclercq and S. Cnockaert, Parity violating vertices for spin-3 gauge fields, Phys. Rev. D 73 (2006) 065019 [hep-th/0509118] [InSPIRE].

[25] N. Boulanger, S. Leclercq and P. Sundell, On The Uniqueness of Minimal Coupling in Higher-Spin Gauge Theory, JHEP 08 (2008) 056 [arXiv:0805.2764] [INSPIRE].

[26] A. Fotopoulos and M. Tsulaia, Gauge Invariant Lagrangians for Free and Interacting Higher Spin Fields. A Review of the BRST formulation, Int. J. Mod. Phys. A 24 (2009) 1 [arXiv:0805.1346] [INSPIRE].

[27] X. Bekaert, N. Boulanger and S. Leclercq, Strong obstruction of the Berends-Burgers-van Dam spin-3 vertex, J. Phys. A 43 (2010) 185401 [arXiv:1002.0289] [InSPIRE].

[28] A. Fotopoulos and M. Tsulaia, On the Tensionless Limit of String theory, Off - Shell Higher Spin Interaction Vertices and BCFW Recursion Relations, JHEP 11 (2010) 086 [arXiv: 1009.0727] [INSPIRE].

[29] R.R. Metsaev, BRST-BV approach to cubic interaction vertices for massive and massless higher-spin fields, Phys. Lett. B 720 (2013) 237 [arXiv:1205.3131] [INSPIRE].

[30] M. Henneaux, G. Lucena Gómez and R. Rahman, Higher-Spin Fermionic Gauge Fields and Their Electromagnetic Coupling, JHEP 08 (2012) 093 [arXiv: 1206.1048] [INSPIRE].

[31] M. Henneaux, G. Lucena Gómez and R. Rahman, Gravitational Interactions of Higher-Spin Fermions, JHEP 01 (2014) 087 [arXiv:1310.5152] [INSPIRE].

[32] D. Francia, J. Mourad and A. Sagnotti, Current Exchanges and Unconstrained Higher Spins, Nucl. Phys. B 773 (2007) 203 [hep-th/0701163] [INSPIRE]. 
[33] D. Francia, J. Mourad and A. Sagnotti, $(A) d S$ exchanges and partially-massless higher spins, Nucl. Phys. B 804 (2008) 383 [arXiv:0803.3832] [InSPIRE].

[34] X. Bekaert, E. Joung and J. Mourad, On higher spin interactions with matter, JHEP 05 (2009) 126 [arXiv:0903.3338] [INSPIRE].

[35] X. Bekaert and E. Meunier, Higher spin interactions with scalar matter on constant curvature spacetimes: conserved current and cubic coupling generating functions, JHEP 11 (2010) 116 [arXiv:1007.4384] [INSPIRE].

[36] R. Manvelyan, R. Mkrtchyan and W. Ruehl, Radial Reduction and Cubic Interaction for Higher Spins in (A)dS space, Nucl. Phys. B 872 (2013) 265 [arXiv:1210.7227] [InSPIRE].

[37] R.R. Metsaev, Poincaré invariant dynamics of massless higher spins: Fourth order analysis on mass shell, Mod. Phys. Lett. A 6 (1991) 359 [INSPIRE].

[38] R.R. Metsaev, $S$ matrix approach to massless higher spins theory. 2: The Case of internal symmetry, Mod. Phys. Lett. A 6 (1991) 2411 [INSPIRE].

[39] M. Taronna, Higher-Spin Interactions: four-point functions and beyond, JHEP 04 (2012) 029 [arXiv: 1107.5843] [INSPIRE].

[40] P. Dempster and M. Tsulaia, On the Structure of Quartic Vertices for Massless Higher Spin Fields on Minkowski Background, Nucl. Phys. B 865 (2012) 353 [arXiv:1203.5597] [INSPIRE].

[41] M.A. Vasiliev, Cubic Vertices for Symmetric Higher-Spin Gauge Fields in $(A) d S_{d}$, Nucl. Phys. B 862 (2012) 341 [arXiv:1108.5921] [INSPIRE].

[42] E. Joung and M. Taronna, Cubic interactions of massless higher spins in (A)dS: metric-like approach, Nucl. Phys. B 861 (2012) 145 [arXiv:1110.5918] [INSPIRE].

[43] E. Joung, L. Lopez and M. Taronna, On the cubic interactions of massive and partially-massless higher spins in (A)dS, JHEP 07 (2012) 041 [arXiv:1203.6578] [INSPIRE].

[44] E. Joung, L. Lopez and M. Taronna, Solving the Noether procedure for cubic interactions of higher spins in (A)dS, J. Phys. A 46 (2013) 214020 [arXiv:1207.5520] [InSPIRE].

[45] M. Taronna, Higher-Spin Interactions: three-point functions and beyond, arXiv:1209.5755 [INSPIRE].

[46] E. Joung, L. Lopez and M. Taronna, Generating functions of (partially-)massless higher-spin cubic interactions, JHEP 01 (2013) 168 [arXiv:1211.5912] [INSPIRE].

[47] E. Joung, M. Taronna and A. Waldron, A Calculus for Higher Spin Interactions, JHEP 07 (2013) 186 [arXiv: 1305.5809] [INSPIRE].

[48] Y. Zinoviev, On massive spin 2 electromagnetic interactions, Nucl. Phys. B 821 (2009) 431 [arXiv:0901.3462] [INSPIRE].

[49] F.A. Berends, G.J.H. Burgers and H. van Dam, On the Theoretical Problems in Constructing Interactions Involving Higher Spin Massless Particles, Nucl. Phys. B 260 (1985) 295 [INSPIRE].

[50] C. Aragone and S. Deser, Consistency Problems of Hypergravity, Phys. Lett. B 86 (1979) 161 [INSPIRE].

[51] X. Bekaert and N. Boulanger, Gauge invariants and Killing tensors in higher-spin gauge theories, Nucl. Phys. B 722 (2005) 225 [hep-th/0505068] [INSPIRE]. 
[52] X. Bekaert, N. Boulanger, S. Cnockaert and S. Leclercq, On Killing tensors and cubic vertices in higher-spin gauge theories, Fortsch. Phys. 54 (2006) 282 [hep-th/0602092] [INSPIRE].

[53] N. Boulanger, D. Ponomarev, E.D. Skvortsov and M. Taronna, On the uniqueness of higher-spin symmetries in AdS and CFT, Int. J. Mod. Phys. A 28 (2013) 1350162 [arXiv: 1305.5180] [INSPIRE].

[54] M.A. Vasiliev, Multiparticle extension of the higher-spin algebra, Class. Quant. Grav. 30 (2013) 104006 [arXiv:1212.6071] [INSPIRE].

[55] O.A. Gelfond and M.A. Vasiliev, Operator algebra of free conformal currents via twistors, Nucl. Phys. B 876 (2013) 871 [arXiv:1301.3123] [INSPIRE].

[56] A. K. Bengtsson, A Unified Action For Higher Spin Gauge Bosons From Covariant String Theory, Phys. Lett. B 182 (1986) 321.

[57] M. Henneaux and C. Teitelboim, quantum mechanics of fundamental systems, C. Teitelboim and J. Zanelli eds., Plenum Press, New York, pg. 113, 1988.

[58] G. Bonelli, On the tensionless limit of bosonic strings, infinite symmetries and higher spins, Nucl. Phys. B 669 (2003) 159 [hep-th/0305155] [INSPIRE].

[59] A. Sagnotti and M. Tsulaia, On higher spins and the tensionless limit of string theory, Nucl. Phys. B 682 (2004) 83 [hep-th/0311257] [INSPIRE].

[60] D. Francia, String theory triplets and higher-spin curvatures, Phys. Lett. B 690 (2010) 90 [arXiv: 1001.5003] [INSPIRE].

[61] A. Campoleoni and D. Francia, Maxwell-like Lagrangians for higher spins, JHEP 03 (2013) 168 [arXiv: 1206.5877] [INSPIRE].

[62] D. Francia, S.L. Lyakhovich and A.A. Sharapov, On the gauge symmetries of Maxwell-like higher-spin Lagrangians, Nucl. Phys. B 881 (2014) 248 [arXiv: 1310.8589] [INSPIRE].

[63] S.R. Coleman and J. Mandula, All Possible Symmetries of the S Matrix, Phys. Rev. 159 (1967) 1251 [INSPIRE].

[64] A.Y. Segal, Conformal higher spin theory, Nucl. Phys. B 664 (2003) 59 [hep-th/0207212] [INSPIRE].

[65] X. Bekaert, E. Joung and J. Mourad, Effective action in a higher-spin background, JHEP 02 (2011) 048 [arXiv: 1012.2103] [INSPIRE].

[66] E. Joung and K. Mkrtchyan, A note on higher-derivative actions for free higher-spin fields, JHEP 11 (2012) 153 [arXiv: 1209.4864] [INSPIRE].

[67] S. Weinberg, Photons and Gravitons in s Matrix Theory: Derivation of Charge Conservation and Equality of Gravitational and Inertial Mass, Phys. Rev. B 135 (1964) 1049.

[68] M. Porrati, Universal Limits on Massless High-Spin Particles, Phys. Rev. D 78 (2008) 065016 [arXiv: 0804.4672] [inSPIRE].

[69] J.M. Martin-Garcia et al., xAct: Efficient tensor computer algebra for Mathematica, 2002-2013.

[70] T. Nutma, xTras: a field-theory inspired xAct package for Mathematica, arXiv:1308.3493 [INSPIRE]. 